\title{
Long-term Non-Invasive Ventilation in Infants: A Systematic Review and Meta-Analysis
}

\author{
Prabhjot K. Bedi', Maria Luisa Castro-Codesal', Robin Featherstone', \\ Mohammed M. AlBalawi ${ }^{3}$, Bashar Alkhaledi ${ }^{4}$, Anita L. Kozyrskyj ${ }^{1,5}$, Carlos Flores-Mir ${ }^{6}$ \\ and Joanna E. MacLean ${ }^{1,5 *}$
}

'Department of Pediatrics, Faculty of Medicine and Dentistry, University of Alberta, Edmonton, AB, Canada, ${ }^{2}$ Alberta Research Centre for Health Evidence, University of Alberta, Knowledge Translation Platform, Alberta SPOR SUPPORT Unit, Edmonton, AB, Canada, ${ }^{3}$ Department of Pediatrics, King Faisal Specialist Hospital and Research Centre, Riyadh, Saudi Arabia, ${ }^{4}$ Pediatric Pulmonary and Sleep Unit, Al-Sabah Hospital, Kuwait City, Kuwait, ${ }^{5}$ Women and Children's Health Research Institute, University of Alberta, Edmonton, AB, Canada, ${ }^{6}$ Faculty of Medicine and Dentistry, School of Dentistry, University of Alberta, Edmonton, $A B$, Canada

Background: The use of long-term non-invasive ventilation (NIV) to treat sleep and breathing disorders in children has increased substantially in the last decade; however, less data exist about its use in infants. Given that infants have distinct sleep and breathing patterns when compared to older children, the outcomes of infants on long-term NIV may differ as well. The aim of this study is to systematically review the use and outcomes of long-term NIV in infants.

Methods: Ovid Medline, Ovid Embase, CINAHL (via EbscoHOST), PubMed, and Wiley Cochrane Library were systematically searched from January 1990 to July 2017. Studies on infants using long-term NIV outside of an acute care setting were included. Data were extracted on study design, population characteristics, and NIV outcomes.

Results: A total of 327 studies were full-text reviewed, with final inclusion of 60. Studies were distributed across airway (40\%), neuromuscular (28\%), central nervous system (10\%), cardio-respiratory (2\%), and multiple (20\%) disease categories. Of the 18 airway studies reporting on NIV outcomes, 13 (72\%) reported improvements in respiratory parameters. Of the 12 neuromuscular studies exclusively on spinal muscular atrophy type 1 (SMA1), six (50\%) reported decreased hospitalizations and nine (75\%) reported on mortality outcomes. Risk of bias was moderate to serious, and quality of the evidence was low to very low for all studies. Most studies had an observational design with no control group, limiting the potential for a meta-analysis.

Conclusion: The outcomes reported in studies differed by the disease category being studied. Studies on airway conditions showed improvements in respiratory parameters for infants using NIV. Studies on neuromuscular disorder, which were almost exclusively on SMA1, reported decreased hospitalizations and prolonged survival. Overall, it appears that NIV is an effective long-term therapy for infants. However, the high risk of bias and low quality of the available evidence limited strong conclusions.

Keywords: continuous positive airway pressure, bi-level positive airway pressure, obstructive sleep apnea, Pierre Robin sequence, laryngo-tracheomalacia, spinal muscular atrophy type 1, central hypoventilation syndrome 


\section{INTRODUCTION}

\section{Rationale}

Long-term non-invasive ventilation (NIV), defined as respiratory support delivered through an interface outside the airway, has become the treatment of choice for a number of chronic conditions resulting in respiratory insufficiency or sleep and breathing disorders in infants and children (1-3). These conditions include airway disorders, neuromuscular disorders (NMDs), and disorders of the central nervous system (CNS) (3-6). The shift toward NIV therapies may have been driven by improvements in NIV technology, a greater emphasis on home-based care, and a growing acceptance of NIV as a viable long-term respiratory support $(1,6,7)$. With the increasing number of infants and children living at home using NIV, understanding the benefits and risks of NIV is becoming important not only for specialists involved in starting this therapy but also for pediatricians and primary care physicians providing care to these children within the community and policy makers responsible for decisions about provision of healthcare resources.

While there is a considerable body of work describing the use of long-term NIV, including continuous positive airway pressure (CPAP) and bi-level positive airway pressure (BPAP), in a broad range of pediatric populations, less is known about its use in infants $(8-10)$. Without sufficient data to suggest otherwise, similar NIV treatment approaches are likely followed in both infants and older children, despite key physiological differences in sleep and breathing patterns in infancy. Both sleep and breathing processes are immature at birth and continue to develop through infancy, resulting in change in sleep patterns and breathing control that continue through early life (11). Sleep occupies a greater proportion of time in infants compared to older children (12), which makes infants more vulnerable to respiratory disorders that disrupt sleep. Immaturity of central respiratory centers in infants contributes to increased respiratory events and a greater variability in oxygen saturation, both of which may be important for the normal development of respiratory control $(11,13)$. Since sleep and breathing processes differ by age, especially in early life, the type of respiratory and sleep disorders treated with NIV, the response to NIV treatment, and the outcomes for NIV may also differ in infants as compared to older children.

Most data available on long-term NIV use in infants is limited to single-center observational studies with relatively small sample sizes (8). Aggregation of the available data for combined data analysis will improve our understanding of the risks and benefits of NIV therapy in the infant population.

\footnotetext{
Abbreviations: ALTE, acute life-threatening events; BPAP, bi-level positive airway pressure; CHS, central hypoventilation syndrome; CNS, central nervous system; CPAP, continuous positive airway pressure; GRADE, Grading of Recommendations Assessment, Development and Evaluation; LTM, laryngo-tracheomalacia; NIV, non-invasive ventilation; NMD, neuromuscular disorder; OSA, obstructive sleep apnea; PRISMA, Preferred Reporting Items for Systematic Reviews and Meta-Analyses; PRS, Pierre Robin sequence; ROBINS-I, Risk of Bias in NonRandomized Studies of Interventions; SIDS, sudden infant death syndrome; SMA1, spinal muscular atrophy type 1 .
}

\section{Objective}

The objective of this systematic review is to summarize the available evidence on the use of long-term NIV for infants and to estimate effect sizes for specific sub-populations and clinical outcomes compared to alternative respiratory care strategies.

\section{Research Question}

Does the use of NIV, compared to supportive care, or invasive ventilation, improve clinical outcomes for infants under the age of 2 years with chronic conditions resulting in respiratory insufficiency or sleep and breathing disorders?

\section{METHODS}

\section{Study Design}

This review was conducted using systematic review methodology.

\section{Participants}

The inclusion criteria for this systematic review were as follows: (1) infants, defined by the Public Health Agency of Canada as ages 0-24 months inclusive (14); (2) NIV use, defined as breathing support delivered from outside the airway; and (3) long-term NIV use, defined as greater than three months outside of an acute care setting. For studies that examined a broader age range, the mean age of NIV initiation had to be less than 24 months in order to be included in this review, or data had to be presented separately for infants. We did not place any restrictions on study design or outcome eligibility.

\section{Systematic Review Protocol}

The protocol for this systematic review was developed according to the Preferred Reporting Items for Systematic Review and Meta-Analyses (PRISMA) guidelines (15). The full protocol has been registered in the PROSPERO database for international prospective reviews (16).

\section{Search Strategy}

This systematic review is an extension of a prior scoping review on long-term NIV in children (8). The scoping review search strategy, using Medical Subject Headings ( $\mathrm{MeSH})$ and free-text terms for "child" and "non-invasive ventilation," was developed for MEDLINE (Ovid) and adapted for subsequent electronic databases with the full protocol published elsewhere (17) [see Table 1 for original MEDLINE (Ovid) search strategy]. Human studies published from 1990 onward were searched in MEDLINE (Ovid), Embase (Ovid), CINAHL (Ebsco), Cochrane Library (Wiley), and PubMed between November 17 and 28, 2014, with no restriction on study design. Gray literature, in the form of conference abstracts on respiratory and sleep medicine, was identified from 2012 to 2014 . The literature search was re-run on April 29, 2016, and July 12, 2017, using the same search strategy in Ovid MEDLINE, Ovid Embase, CINAHL, and Wiley Cochrane Library to identify additional studies. 
TABLE 1 | Search strategy used in the Ovid Medline database for the scoping review to identify literature on the use of long-term non-invasive ventilation in children.

Ovid MEDLINE(R) In-Process and other non-indexed citations and Ovid Medline(R): 1946 to November Week 1, 2014

Original search date: 17 November 2014

Update search dates: 29 April 2016 and 12 July 2017

1. Continuous Positive Airway Pressure/

2. Noninvasive Ventilation/

3. Intermittent Positive-Pressure Breathing/

4. Ventilators, Negative-Pressure/

5. AVAPS.tw.

6. [(auto* or adaptive) adj2 (servoventilation or ventilation)].tw.

7. AutoSet ${ }^{\star}$.tw.

8. ((bi level or bi-level) adj2 (airway ${ }^{\star}$ or air way ${ }^{\star}$ or assist ${ }^{\star}$ or breath* or positive pressure ${ }^{\star}$ or

respirat $^{\star}$ or ventilat ${ }^{\star}$ or support ${ }^{\star}$ or therap $\left.{ }^{\star}\right)$ ).tw.

9. BIPAP*.tw.

10. BPAP*.tw.

11. c flex.tw.

12. CNEP.tw.

13. (continuous negative adj2 pressure).tw.

14. (continuous positive airway* or continuous positive air way*).tw.

15. (continuous positive adj2 pressure).tw.

16. CPAP $^{\star}$.tw.

17. ((domicil ${ }^{\star}$ or home $\left.{ }^{\star}\right)$ adj5 ventilat $\left.{ }^{\star}\right)$.tw.

18. intermittent positive pressure breathing.tw.

19. IPPB $^{\star}$.tw.

20. ((long term or longterm) adj5 ventilat $\left.{ }^{\star}\right)$.tw.

21. ((nasal ${ }^{*}$ or mask $^{*}$ ) adj2 (positive adj2 pressure)).tw.

22. ((nasal ${ }^{*}$ or mask $\left.^{*}\right)$ adj2 ventilat $\left.{ }^{\star}\right)$.tw.

23. nCPAP*.tw

24. ((negative pressure) adj2 (respirat* or ventilat $\left.\left.{ }^{\star}\right)\right)$.tw.

25. ((night ${ }^{*}$ or nocturnal ${ }^{*}$ or sleep $\left.{ }^{\star}\right)$ adj5 ventilat*).tw.

26. NIPPV*.tw.

27. ((noninvasive adj5 ventilat*) or (non invasive adj5 ventilat*)).tw.

28. (noninvasive respiratory support ${ }^{\star}$ or non invasive respiratory support*).tw.

29. NPPV*.tw.

30. (positive pressure adj2 respirat $\left.{ }^{\star}\right)$.tw.

31. REMstar*.tw.

32. (tank adj (respirat* or ventilat*)).tw.

33. VPAP*.tw.

34. or/1-33

35. Hypoventilation/pc, rh, th [Prevention \& Control, Rehabilitation, Therapy]

36. Interactive Ventilatory Support/

37. Intermittent Positive-Pressure Ventilation/

38. Positive-Pressure Respiration/

39. Respiration, Artificial/

40. Respiratory Insufficiency/pc, rh, th [Prevention \& Control, Rehabilitation, Therapy]
41. exp Sleep Apnea Syndromes/pc, rh, th [Prevention \& Control,

Rehabilitation, Therapy]

42. Ventilators, Mechanical/

43. ((airway* or air way ${ }^{\star}$ or breath* or inspirat* or respirat* or ventilat*)

and (positive adj2 pressure)).tw.

44. intermittent positive pressure.tw.

45. IPPV*.tw.

46. (mechanical adj (respirat ${ }^{\star}$ or ventilat $\left.\left.{ }^{\star}\right)\right)$. tw.

47. (positive adj2 pressure adj (assist ${ }^{\star}$ or support ${ }^{\star}$ or therap $\left.{ }^{\star}\right)$ ).tw.

48. positive airway pressure.tw.

49. pulmonary ventilator ${ }^{\star}$.tw.

50. respiratory support ${ }^{\star} . t w$.

51. or/35-50

52. (noninvasive or non invasive or spontaneous*).mp.

53. 51 and 52

54. 34 or 53

55. exp Adolescent/

56. exp Child/

57. exp Infant/

58. exp Minors/

59. exp Pediatrics/

60. exp Puberty/

61. exp Schools/

62. adoles*.mp.

63. (baby ${ }^{\star}$ or babies or infant* or infancy or neonat ${ }^{\star}$ or newborn ${ }^{\star}$ or postmatur $^{\star}$ or prematur ${ }^{\star}$ or preterm*).mp.

64. $\left(\right.$ boy $^{\star}$ or girl ${ }^{\star}$ or teen $\left.{ }^{\star}\right) \cdot \mathrm{mp}$.

65. (child* or kid or kids or preschool* or school age* or schoolchild* or toddler $)^{\star} \cdot \mathrm{mp}$.

66. (elementary school ${ }^{\star}$ or high school ${ }^{\star}$ or highschool ${ }^{\star}$ or kindergar $^{\star}$ or nursery school* or primary school ${ }^{*}$ or secondary school*).mp.

67. minors*.mp.

68. (pediatric ${ }^{\star}$ or peadiatric ${ }^{\star}$ or pediatric*).mp.

69. (prepubescen ${ }^{\star}$ or pubescen ${ }^{\star}$ or pubert $\left.{ }^{\star}\right) \cdot \mathrm{mp}$.

70. or/55-69

71.54 and 70

72. (case reports or comment or editorial or letter).pt.

73. 71 not 72

74. exp animals/not humans.sh.

75. 73 not 74

76. limit 75 to $y r=" 1990-C u r r e n t "$

77. remove duplicates from 76

The search strategy also included infant keywords to help identify studies on infants.

\section{Data sources, Study Selection, and Data Extraction}

The titles and abstracts of studies identified by the literature search were screened by two reviewers (JEM and MCC) to determine eligibility for full-text retrieval. English, French, Spanish, and Portuguese studies that were considered eligible were full-text reviewed for inclusion by two reviewers (JEM and MCC). The final included studies pertaining to children 0-18 years were then full-text screened by two reviewers (PKB and MMA) to identify studies relevant to infants for inclusion in this systematic review. Any disagreement at the screening, eligibility, and inclusion levels were discussed until a consensus was reached. The reference lists of studies meeting inclusion were also reviewed to identify any additional relevant literature.

Data were entered into a pre-established data collection form in Microsoft Excel (version 14.0.4760, Microsoft Corporation, 2010). These data included author's name, year of publication, country of publication, study design, sample size, age of NIV initiation, NIV type, primary underlying disease conditions, comorbidities, and primary and secondary outcome measures. One reviewer (PKB) extracted the data, and $20 \%$ of data extraction was verified by a second reviewer (MCC).

\section{Risk of Bias}

The Cochrane Risk of Bias in Non-Randomized Studies of Interventions (ROBINS-I) tool (18) was used to assess the risk 
of bias in individual studies. The tool measured confounding, selection, measurement, missing data, and reporting bias. Bias was ranked as low, moderate, severe, critical, or no information. Risk of bias in individual studies was independently assessed by two reviewers (PKB and MMA), with disagreements resolved by discussion and consensus.

\section{Quality Assessment}

The Grading of Recommendations Assessment, Development and Evaluation (GRADE) tool (19) was used to determine the quality of studies at an outcome level. Two reviewers (PKB and MMA) independently assessed the quality of studies, with disagreements being resolved through discussion and consensus. Meta-analysis was performed to calculate risk ratios for appropriate outcomes using Review Manager (version 5.3., Copenhagen: The Nordic Cochrane Centre, The Cochrane Collaboration, 2014).

\section{Synthesis of Results}

Studies were grouped by disease category (airway, NMD, CNS, cardio-respiratory or multiple disorders) after the data collection stage, to allow for adequate pathophysiological comparisons. Within each disease category, studies were grouped based on primary disease conditions. We included studies with infants who had multiple disease conditions under one disease heading if $>75 \%$ of the infant cohort had the same disease condition; otherwise these studies were included in the multiple disorders category.

Primary and secondary outcomes were established after data collection, during synthesis of the data, based on the most common and clinically relevant outcomes reported in studies with the same disease condition. Primary outcomes were as follows: (1) objective changes in respiratory parameters, (2) discontinuation of NIV, (3) hospitalizations, and (4) mortality. Secondary outcomes were as follows: (1) improvements in underlying disease conditions, (2) improvements in growth parameters, (3) NIV facilitation of extubation, (4) predictors of NIV requirement, (5) NIV success/failure, (6) adherence to respiratory support, and (7) mask complications. Studies were included in the synthesis if they reported on at least one primary or secondary outcome. Continuous data were presented as a weighted mean (standard deviation) or median (interquartile range) where appropriate. Results were grouped and reported based on the primary underlying disease category being studied. Primary outcomes were reported in both tabular and narrative format, while secondary outcomes were only reported narratively.

\section{RESULTS}

\section{Study Selection and Characteristics}

The search strategy, after removal of duplicates, identified 12,594 studies and additional records (Figure 1). After screening of the titles and abstracts, and with the addition of records from additional sources, 1046 studies met eligibility for review. After full-text review, 327 studies on children ages 0-18 years met the inclusion criteria for the scoping review. Full-text review of these 327 articles identified 64 studies meeting the infant inclusion criteria. Four conference proceedings met inclusion criteria but were excluded because of insufficient data reporting, leaving 60 articles reporting on a total of 977 infants for inclusion in this systematic review (Table 2) (3, 7, 9, 10, 20-75).

The majority of studies were retrospective $(41 / 60,68 \%)$, quantitative $(59 / 60,98 \%)$, and single-center studies (54/60, $90 \%)$. The most common study design was observational, which included cohort studies $(31 / 60,52 \%)$, case series $(13 / 60,25 \%)$, and cross-sectional studies $(8 / 60,13 \%)$. Forty-eight percent of studies were exclusively on the infant population. Based on primary underlying disease categories, the studies were distributed across airway disorders (24/60, 40\%), NMD (17/60, 28\%), CNS $(6 / 60,10 \%)$, cardio-respiratory diseases $(1 / 60,2 \%)$, and multiple disease categories (12/60, 20\%; Table 2). Thirteen studies did not report NIV outcomes, only the number of infants using NIV, and were excluded from further analysis $(7,25,26,32,33,37,47,52$, $57,59,72,74,75)$.

\section{Obstructive Sleep Apnea}

Obstructive sleep apnea (OSA) was the most common airway disorder studied in the infant population, with 12 studies $(12 / 60$, $20 \%$ ) reporting on this condition (Table 2). Of these, 10 studies reported on infant NIV outcomes and were synthesized in the review $(10,20-24,27-30)$. These studies included infants with multiple underlying conditions, the most common being a history of acute life-threatening events (ALTE), family history of sudden infant death syndrome (SIDS), and craniofacial malformations. Eight studies $(8 / 10,80 \%)$ reported on changes in respiratory parameters, with seven of these studies (7/10, 70\%) showing improvements in central, obstructive, and/or mixed apneas from a diagnostic to titration polysomnography (Table 3) (10, 20, 22, $23,27-29)$ Only one study $(1 / 10,10 \%)$ included diagnostic polysomnography results after long-term NIV use (weighted mean of 12 months), which showed an overall decrease in respiratory events, normalization of respiratory gases, and increased arousals during REM sleep (29). Five studies (5/10, 50\%) reported discontinuation of NIV in infants because of improvements in respiratory parameters, with discontinuation rates ranging from 14 to $100 \%$ (weighted mean $70 \pm 26 \%)(20,21,27,29,30)$. No studies reported on hospitalization outcomes (Table 4). One study (1/10, $10 \%$ ) of five infants using NIV reported mortality outcomes, with all infants alive at the time of study publication (27).

\section{Pierre Robin Sequence}

Seven studies $(7 / 60,12 \%)$ reported on infants with Pierre Robin sequence (PRS) using long-term NIV (Table 2). Four studies $(4 / 7,57 \%)$ reported on primary or secondary outcomes and were synthesized for this review $(31,34-36)$. A cohort study reported normalization of polygraphy parameters and gas exchange postNIV initiation (Table 3) (31). A case series reported a decrease in respiratory rates, statistically significant improvements in respiratory effort, and normalization of respiratory gases after administration of NIV therapy in infants with PRS (36). Two studies on 16 infants with PRS reported discontinuation from NIV in 11 (69\%) infants because of improvements in respiratory parameters $(31,36)$. Two studies comparing infants on NIV and invasive mechanical ventilation showed that the length of hospitalization were shorter for infants on NIV than for those receiving invasive mechanical ventilation via a tracheostomy (Table 4) (31, 


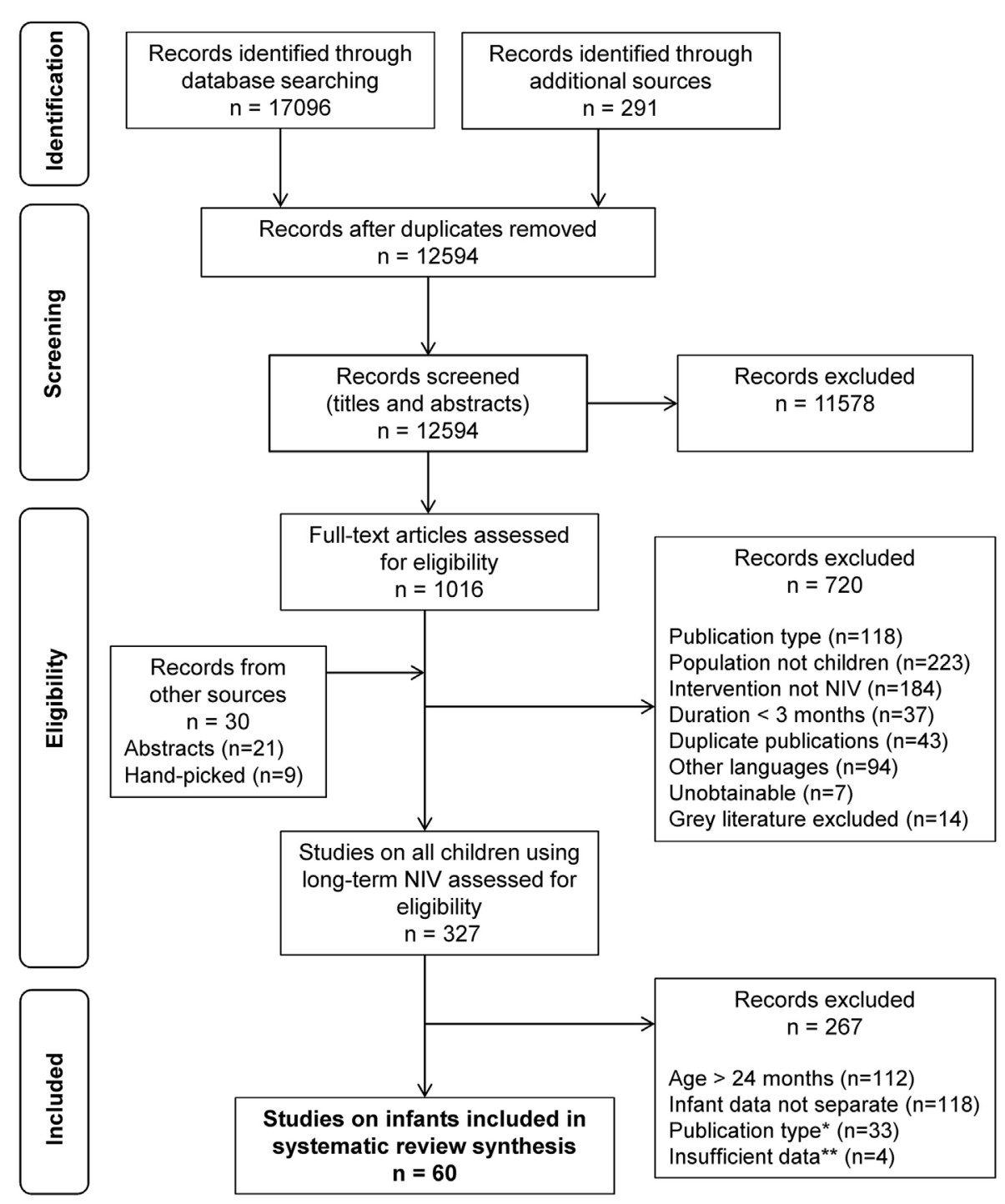

* Case studies or articles with $<2$ infants on NIV or bench studies not on infants.

${ }^{* *}$ There were four abstracts that had insufficient data on infant NIV use to be included in the review.

FIGURE 1 | Flow diagram outlining the study selection process for the systematic review, following the Preferred Reporting Items for Systematic Reviews and Meta-Analyses guidelines (15).

35). No studies addressed survival outcomes in infants with PRS using long-term NIV. Adherence of infants to NIV was reported as excellent, showing more than 8 hours of NIV use per day in two studies $(31,36)$, with only a $1-2$ week period required to adjust to the mask ventilation $(31,35)$. An additional cohort study demonstrated that infants with PRS using NIV were 10.43 times more likely to progress to a surgical airway compared to infants who required less advanced respiratory supports such as prone positioning and a nasopharyngeal airway (34).

\section{Laryngo-Tracheomalacia}

All four studies (4/60,7\%) on infants with laryngo-tracheomalacia (LTM) using long-term NIV reported on primary or secondary outcomes and were synthesized in the review (Table 2) (38-41). Three studies $(3 / 4,75 \%)$ reported on changes in respiratory parameters (Table 3) $(38,39,41)$. A case-control study of 10 infants with LTM showed improvements in respiratory frequency and respiratory effort in infants using CPAP or BPAP compared to spontaneous breathing (38). Normalization of arterial oxygen saturations after NIV use was seen in two studies $(39,41)$. NIV discontinuation was reported in two studies, with a combined discontinuation due to improvement rate of $81 \%$ (13/16 infants) $(39,40)$. No studies examined hospitalization or mortality outcomes. Improvement in chest wall deformity after NIV use in three patients and normalization of weight in four patients was reported in one case-control study (39). The same study 
TABLE 2 | Characteristics and outcomes of 60 studies included in the systematic review on infants using long-term NIV.

\begin{tabular}{|c|c|c|c|c|c|}
\hline $\begin{array}{l}\text { First author, year, } \\
\text { country }\end{array}$ & Study design & Study duration & Total $n(\mathrm{M} / \mathrm{F})$ & Infants on NIV & $\begin{array}{l}\text { Age [mean } \pm \text { SD } \\
\text { or median (range) } \\
\text { unless otherwise }\end{array}$ \\
\hline
\end{tabular}

Interventions

Infant NIV outcomes

stated]

\section{Articles on airway disorders: obstructive sleep apnea}

Downey (20), 2000, Quantitate:

USA

Quantitative:

7 years

18 (n/a)

$n=10^{\text {a }}$

Overall: <2 years

$\operatorname{CPAP}(n=14)$
$\operatorname{IMV}(n=4)$

in respi-

ratory parameters

- Discontinuation

of NIV

Guilleminault (21),
1995, USA

Quantitative:

n/a

$74(35 / 39)$

$n=74$

$24 \pm 9$ weeks

CPAP $(n=74)$

Discontinuation

of NIV

- Changes in respi-

ratory parameters

Harrington (22), 2003,

Quantitative:

$\mathrm{n} / \mathrm{a}$

$18(11 / 7)$

$n=6$

$13 \pm 4$ weeks

$\operatorname{CPAP}(n=6)$ (case-control)

\begin{tabular}{ll}
\hline Leonardis (23), 2013, & Quantitative: \\
USA & observational
\end{tabular}

observational

4 years

$126(86 / 40)$

$n=18$

NIV group: 16 months

None $(n=33)$

NIV $(n=18)$

- Changes in respi-

ratory parameters

- Number of sub-

(cross-sectional)

Liu (24), 2012, China Quantitative:

$\mathrm{n} / \mathrm{a}$

$3(2 / 1)$

MV $(n=7)^{\circ}$

observational (case

Overall: 1 month to

$\operatorname{CPAP}(n=2)$

series)

5 years

$\operatorname{BPAP}(n=2)$

- Changes in respi-

ratory parameters

- Number of sub-

jects on NIV

- Benefit of

NIV (growth

parameters)

Marcus (25), 1995,

\section{Quantitative:}

$\mathrm{n} / \mathrm{a}$

$94(60 / 34)$

$n=3^{\circ}$

Overall: $<1-19$ years $\operatorname{CPAP}(n=94)$

(cross-sectional)

infants: $<1$ year

- Number of sub-

jects on $\mathrm{NIV}^{\star}$

Massa (26), 2002, UK Quantitative:

observational (cohort)

5 years

$66(39 / 27)$

$(n=3)$

observational (cohort)

McNamara (27), Quantitative: control $\quad 0.5$ years

CPAP $(n=66)$

$5.9 \pm 5.1$ years

Infants: $<1$ year

$(n=18)$

1995, Australia before-after

$5(2 / 3)$

$8-12$ weeks

$n=5$

8.

(

(1)

Changes in respi-
ratory parameters

ratory parameters

of NIV

- Survival/mortality

McNamara (28),

Quantitative:

observational

$24(13 / 11)$

$n=8$

CPAP group:

$\operatorname{CPAP}(n=8)$

- Changes in respi-

ratory parameters

- Discontinuation

of NIV

McNamara (29)

Quantitative:

$n / a$

$24(15 / 9)$

$n=24$

1-51 weeks

$\operatorname{CPAP}(n=24)$

Changes in respi-

ratory parameters

Number of sub-
jects on NIV 


\begin{tabular}{|c|c|c|c|c|c|c|c|c|}
\hline \multirow{2}{*}{$\begin{array}{l}\text { First author, year, } \\
\text { country }\end{array}$} & \multirow[t]{2}{*}{ Study design } & \multirow[t]{2}{*}{ Study duration } & \multirow[t]{2}{*}{ Total $n(M / F)$} & \multirow[t]{2}{*}{ Infants on NIV } & \multirow{2}{*}{$\begin{array}{l}\text { Age [mean } \pm \text { SD } \\
\text { or median (range) } \\
\text { unless otherwise } \\
\text { stated] }\end{array}$} & \multirow[t]{2}{*}{ Interventions } & \multicolumn{2}{|c|}{ Infant NIV outcomes } \\
\hline & & & & & & & Primary & Secondary \\
\hline $\begin{array}{l}\text { Robison (10), 2013, } \\
\text { USA }\end{array}$ & $\begin{array}{l}\text { Quantitative: } \\
\text { observational } \\
\text { (cross-sectional) }\end{array}$ & 4 years & $295(196 / 99)$ & $n=18$ & $\begin{array}{l}\text { CPAP/bi-level } \\
\text { group: } 15.6 \text { months } \\
\text { (3-29 months) }\end{array}$ & $\begin{array}{l}\text { None }(n=76) \\
\text { NIV }(n=18) \\
\text { T\&A }(n=116) \\
\operatorname{IMV}(n=6)^{b}\end{array}$ & $\begin{array}{l}\text { - Changes in respi- } \\
\text { ratory parameters }\end{array}$ & $\begin{array}{l}\text { - Number of sub- } \\
\text { jects on NIV }\end{array}$ \\
\hline $\begin{array}{l}\text { Rosen (30), 2010, } \\
\text { USA }\end{array}$ & $\begin{array}{l}\text { Quantitative: } \\
\text { observational (case } \\
\text { series) }\end{array}$ & 5.5 years & $16(n / a)$ & $n=6$ & Overall: <2 years & $\operatorname{CPAP}(n=6)$ & $\begin{array}{l}\text { - Discontinuation } \\
\text { of NIV }\end{array}$ & $\begin{array}{l}\text { - Number of sub- } \\
\text { jects on NIV }\end{array}$ \\
\hline \multicolumn{9}{|c|}{ Articles on airway disorders: Pierre Robin sequence } \\
\hline $\begin{array}{l}\text { Amaddeo (31), 2016, } \\
\text { France }\end{array}$ & $\begin{array}{l}\text { Quantitative: } \\
\text { observational (cohort) }\end{array}$ & 1 year & $44(\mathrm{n} / \mathrm{a})$ & $n=9$ & Infants: 0-2 months & $\operatorname{CPAP}(n=9)$ & $\begin{array}{l}\text { - Changes in respi- } \\
\text { ratory parameters } \\
\text { - Discontinuation } \\
\text { of NIV } \\
\text { - Hospitalizations }\end{array}$ & $\begin{array}{l}\text { - Number of sub- } \\
\text { jects on NIV } \\
\text { - Adherence to NIV }\end{array}$ \\
\hline $\begin{array}{l}\text { Cheng (32), 2011, } \\
\text { Australia }\end{array}$ & $\begin{array}{l}\text { Quantitative: } \\
\text { observational (case } \\
\text { series) }\end{array}$ & 5 years & $6(\mathrm{n} / \mathrm{a})$ & $n=6$ & 26 days to 11 months & $\operatorname{CPAP}(n=6)$ & & $\begin{array}{l}\text { - Number of sub- } \\
\text { jects on NIV* }\end{array}$ \\
\hline $\begin{array}{l}\text { Daniel (33), 2013, } \\
\text { Australia }\end{array}$ & $\begin{array}{l}\text { Quantitative: } \\
\text { observational } \\
\text { (cross-sectional) }\end{array}$ & 12 years & $39(16 / 23)$ & $n=18$ & $n / a$ & $\operatorname{CPAP}(n=18)$ & & $\begin{array}{l}\text { - Number of sub- } \\
\text { jects on } \mathrm{NIV}^{*}\end{array}$ \\
\hline $\begin{array}{l}\text { Goudy (34), 2017, } \\
\text { USA }\end{array}$ & $\begin{array}{l}\text { Quantitative: } \\
\text { observational (cohort) }\end{array}$ & 9 years & $38(18 / 20)$ & $n=9$ & n/a (neonates) & $\begin{array}{l}\operatorname{NIV}(n=9) \\
\operatorname{NPA}(n=14) \\
\operatorname{IMV}(n=8) \\
\operatorname{MDO}(n=5)\end{array}$ & & $\begin{array}{l}\text { - Number of sub- } \\
\text { jects on NIV } \\
\text { - NIV success/failure }\end{array}$ \\
\hline $\begin{array}{l}\text { Kam (35), 2015, } \\
\text { Canada }\end{array}$ & $\begin{array}{l}\text { Quantitative: } \\
\text { observational (cohort) }\end{array}$ & 11 years & $139(72 / 67)$ & $n=20^{d}$ & $\begin{array}{l}23 \text { months ( } 5 \text { days to } \\
8 \text { years) }\end{array}$ & $\begin{array}{l}\text { None }(n=61) \\
\operatorname{CPAP}(n=20) \\
\operatorname{IMV}(n=19)^{\mathrm{b}}\end{array}$ & - Hospitalizations & $\begin{array}{l}\text { - Number of sub- } \\
\text { jects on NIV }\end{array}$ \\
\hline $\begin{array}{l}\text { Leboulanger (36), } \\
\text { 2010, France }\end{array}$ & $\begin{array}{l}\text { Quantitative: } \\
\text { observational (case } \\
\text { series) }\end{array}$ & 10 years & $7(3 / 4)$ & $n=7$ & $1-10$ months & $\begin{array}{l}\operatorname{CPAP}(n=5) \\
\operatorname{BPAP}(n=2)\end{array}$ & $\begin{array}{l}\text { - Changes in respi- } \\
\text { ratory parameters } \\
\text { - Discontinuation } \\
\text { of NIV }\end{array}$ & $\begin{array}{l}\text { - Number of sub- } \\
\text { jects on NIV } \\
\text { - Adherence to NIV }\end{array}$ \\
\hline $\begin{array}{l}\text { Müller-Hagedorn (37), } \\
\text { 2017, Germany }\end{array}$ & $\begin{array}{l}\text { Quantitative: } \\
\text { observational (cohort) }\end{array}$ & 7 years & $68(\mathrm{n} / \mathrm{a})$ & $n=5$ & $\mathrm{n} / \mathrm{a}$ & $\operatorname{CPAP}(n=5)$ & & $\begin{array}{l}\text { - Number of sub- } \\
\text { jects on } \mathrm{NIV}^{*}\end{array}$ \\
\hline \multicolumn{9}{|c|}{ Articles on upper airway disorders: Laryngo-tracheomalacia } \\
\hline $\begin{array}{l}\text { Essouri (38), 2005, } \\
\text { France }\end{array}$ & $\begin{array}{l}\text { Quantitative: control } \\
\text { before-after }\end{array}$ & $\mathrm{n} / \mathrm{a}$ & $10(5 / 5)$ & $n=10$ & $\begin{array}{l}9.5 \text { months } \\
\text { (3-18 months) }\end{array}$ & $\begin{array}{l}\text { None }(n=10) \\
\operatorname{CPAP}(n=10) \\
\operatorname{BPAP}(n=10)\end{array}$ & $\begin{array}{l}\text { - Changes in respi- } \\
\text { ratory parameters }\end{array}$ & $\begin{array}{l}\text { - Number of sub- } \\
\text { jects on NIV }\end{array}$ \\
\hline
\end{tabular}




\begin{tabular}{lllll}
\hline $\begin{array}{l}\text { Fauroux (39), 2001, } \\
\text { France, UK }\end{array}$ & $\begin{array}{l}\text { Quantitative: control } \\
\text { before-after }\end{array}$ & n/a & $12(10 / 2)$ & $n=5$
\end{tabular}

Overall: $\quad$ None $(n=12)$

$32.9 \pm 25.8$ months $\operatorname{BPAP}(n=12)$

Infants: 8-19 months

Primary

\section{Secondary}

- Changes in respi- - Number of sub-

ratory parameters

jects on NIV

- Discontinuation - Adherence to NIV

of NIV

- Benefit of

NIV (growth

parameters)

\begin{tabular}{|c|c|c|c|c|c|c|c|c|}
\hline $\begin{array}{l}\text { Shatz (40), 2004, } \\
\text { Israel }\end{array}$ & $\begin{array}{l}\text { Quantitative: } \\
\text { observational (cohort) }\end{array}$ & 3 years & $50(36 / 14)$ & $n=50$ & $\begin{array}{l}6.5 \pm 3.5 \text { months } \\
\text { (1-18 months) }\end{array}$ & $\begin{array}{l}\operatorname{CPAP}(n=5) \\
\operatorname{BPAP}(n=9)\end{array}$ & $\begin{array}{l}\text { - Discontinuation } \\
\text { of NIV }\end{array}$ & $\begin{array}{l}\text { - Number of sub- } \\
\text { jects on NIV } \\
\text { - Improvement in } \\
\text { underlying disease }\end{array}$ \\
\hline $\begin{array}{l}\text { Zwacka (41), 1997, } \\
\text { Germany }\end{array}$ & $\begin{array}{l}\text { Quantitative: } \\
\text { observational (case } \\
\text { series) }\end{array}$ & $\mathrm{n} / \mathrm{a}$ & $10(5 / 5)$ & $n=10$ & 3 weeks to 5 months & $\operatorname{CPAP}(n=7)$ & $\begin{array}{l}\text { - Changes in respi- } \\
\text { ratory parameters }\end{array}$ & $\begin{array}{l}\text { - Number of sub- } \\
\text { jects on NIV } \\
\text { - Benefit of } \\
\text { NIV (growth } \\
\text { parameters) }\end{array}$ \\
\hline
\end{tabular}

Articles on airway disorders: breath holding spells

\begin{tabular}{|c|c|c|c|c|c|c|c|c|}
\hline $\begin{array}{l}\text { Guilleminault (42), } \\
\text { 2007, USA, Taiwan }\end{array}$ & $\begin{array}{l}\text { Quantitative: } \\
\text { observational } \\
\text { (case-control) }\end{array}$ & 2.5 years & $19(11 / 8)$ & $n=14$ & $31 \pm 3$ weeks & $\operatorname{CPAP}(n=14)$ & $\begin{array}{l}\text { - Changes in respi- } \\
\text { ratory parameters }\end{array}$ & $\begin{array}{l}\text { - Number of sub- } \\
\text { jects on NIV } \\
\text { - NIV success/failure }\end{array}$ \\
\hline
\end{tabular}

Articles on neuromuscular disease: spinal muscular atrophy type 1

\begin{tabular}{|c|c|c|c|c|c|c|c|c|}
\hline Bach (43), 2000, USA & $\begin{array}{l}\text { Quantitative: } \\
\text { observational (case } \\
\text { series) }\end{array}$ & $n / a$ & $11(6 / 5)$ & $n=8$ & 3-28 months & $\operatorname{BPAP}(n=11)$ & $\begin{array}{l}\text { - Hospitalizations } \\
\text { - Survival/Mortality }\end{array}$ & $\begin{array}{l}\text { - Number of sub- } \\
\text { jects on NIV } \\
\text { - } \text { Benefit of NIV } \\
\text { (extubation) } \\
\text { - Benefit of } \\
\text { NIV (growth } \\
\text { parameters) }\end{array}$ \\
\hline Bach (44), 2002, USA & $\begin{array}{l}\text { Quantitative: } \\
\text { observational (cohort) }\end{array}$ & 5 years & 56 (n/a) & $n=33$ & $\begin{array}{l}\text { Overall for patient } \\
\text { groups: NIV: } \\
11.2 \pm 5.7 \text { months } \\
\text { IMV: } \\
10.8 \pm 5.0 \text { months } \\
\text { supportive: } \\
6.0 \pm 1.3 \text { months }\end{array}$ & $\begin{array}{l}\text { NIV }(n=33) \\
\text { IMV }(n=16) \\
\text { None }(n=7)\end{array}$ & $\begin{array}{l}\text { - Hospitalizations } \\
\text { - Survival/mortality }\end{array}$ & $\begin{array}{l}\text { - Number of sub- } \\
\text { jects on NIV }\end{array}$ \\
\hline Bach (45), 2003, USA & $\begin{array}{l}\text { Quantitative: } \\
\text { observational (case } \\
\text { series) }\end{array}$ & $\mathrm{n} / \mathrm{a}$ & $3(2 / 1)$ & $n=3$ & 4-11 months & $\operatorname{NIV}(n=3)$ & & $\begin{array}{l}\text { - Number of sub- } \\
\text { jects on NIV } \\
\text { - Benefit of } \\
\text { NIV (growth } \\
\text { parameters) }\end{array}$ \\
\hline
\end{tabular}




\begin{tabular}{llll}
\hline Bach (46), 2007, USA Quantitative: & 13 & $92(n / a)$ & $n=92^{\mathrm{d}}$
\end{tabular}

$$
\text { observational (cohort) }
$$

Therapy group: none:

Therapy group: none:
$6.6 \pm 4.1$ months

bi-level:

$10.6 \pm 5.7$ months

IMV:

$14.8 \pm 15.2$ months

$\begin{array}{ll}\text { Barnerias (47), 2014, } & \begin{array}{l}\text { Quantitative: } \\ \text { France }\end{array} \\ & \text { observational } \\ & \text { (cross-section }\end{array}$

20 years

$222(\mathrm{n} / \mathrm{a})$

Overall: 3 months

(0.5-8 months)

$\operatorname{NIV}(n=8)$

4-9 months

$\operatorname{BPAP}(n=4)$

$4(3 / 1)$

$n=3$

- Survival/mortality

observational (case

2 years

series)

Chatwin (49), 2011 $\quad$ Quantitative:

UK

Quantitative:
observational (cohort)

19 years

$13(8 / 5)$

$n=13$

4-24 months

$\operatorname{BPAP}(n=13)$

- Survival/mortality

jects on NIV

- Benefit of NIV (extubation)

\begin{tabular}{|c|c|c|c|c|c|c|c|c|}
\hline $\begin{array}{l}\text { Ednick (50), 2008, } \\
\text { USA }\end{array}$ & $\begin{array}{l}\text { Quantitative: } \\
\text { observational (cohort) }\end{array}$ & 3.5 years & $7(1 / 6)$ & $n=7$ & $8.3 \pm 3.7$ months & $\operatorname{BPAP}(n=7)$ & & $\begin{array}{l}\text { - Number of sub- } \\
\text { jects on NIV } \\
\text { - Benefit of NIV } \\
\text { (extubation) }\end{array}$ \\
\hline $\begin{array}{l}\text { Gregoretti (51), 2013, } \\
\text { Italy }\end{array}$ & $\begin{array}{l}\text { Quantitative: } \\
\text { observational (case } \\
\text { series) }\end{array}$ & 18 years & $194(103 / 91)$ & $n=31$ & $\begin{array}{l}\text { NIV group: } \\
12.6 \pm 14.4 \text { months } \\
\text { (0-42 months) } \\
\text { IMV group: } \\
6.9 \pm 4.3 \text { months }\end{array}$ & $\begin{array}{l}\text { None }(n=121) \\
\operatorname{NIV}(n=31) \\
\operatorname{IMV}(n=42)\end{array}$ & $\begin{array}{l}\text { - Hospitalizations } \\
\text { - Survival/mortality }\end{array}$ & $\begin{array}{l}\text { - Number of sub- } \\
\text { jects on NIV }\end{array}$ \\
\hline $\begin{array}{l}\text { loos (52), 2004, } \\
\text { France }\end{array}$ & $\begin{array}{l}\text { Quantitative: } \\
\text { observational (cohort) }\end{array}$ & $\mathrm{n} / \mathrm{a}$ & 180 (n/a) & $n=33$ & $19 \pm 17$ months & $\mathrm{n} / \mathrm{a}$ & & $\begin{array}{l}\text { - Number of sub- } \\
\text { jects on } \mathrm{NIV}^{*}\end{array}$ \\
\hline $\begin{array}{l}\text { Lemoine (53), 2012, } \\
\text { USA }\end{array}$ & $\begin{array}{l}\text { Quantitative: } \\
\text { observational (cohort) }\end{array}$ & 7 years & $49(31 / 18)$ & $n=49$ & $\begin{array}{l}\text { Groups: NIV: } \\
136 \text { days } \\
\text { (34-196 days) } \\
\text { Supportive } \\
\text { care: } 69 \text { days } \\
\text { (38-145 days) }\end{array}$ & $\begin{array}{l}\text { None }(n=23) \\
\operatorname{BPAP}(n=26)\end{array}$ & $\begin{array}{l}\text { - Hospitalizations } \\
\text { - Survival/mortality }\end{array}$ & $\begin{array}{l}\text { - Number of sub- } \\
\text { jects on NIV }\end{array}$ \\
\hline $\begin{array}{l}\text { Ottonello (54), 2011, } \\
\text { Italy }\end{array}$ & $\begin{array}{l}\text { Quantitative: } \\
\text { observational (cohort) }\end{array}$ & 4 years & $16(\mathrm{n} / \mathrm{a})$ & $n=14^{e}$ & $\begin{array}{l}\text { Overall: }<3 \text { years } \\
\text { Infants: } \\
10.4 \pm 6.2 \text { months }\end{array}$ & $\operatorname{NIV}(n=16)$ & $\begin{array}{l}\text { - Hospitalizations } \\
\text { - Survival/mortality }\end{array}$ & $\begin{array}{l}\text { - Number of sub- } \\
\text { jects on NIV } \\
\text { - Benefit of NIV }\end{array}$ \\
\hline
\end{tabular}


TABLE 2 | Continued

\begin{tabular}{|c|c|c|c|c|c|c|c|c|}
\hline \multirow{2}{*}{$\begin{array}{l}\text { First author, year, } \\
\text { country }\end{array}$} & \multirow[t]{2}{*}{ Study design } & \multirow[t]{2}{*}{ Study duration } & \multirow[t]{2}{*}{ Total $n(\mathrm{M} / \mathrm{F})$} & \multirow[t]{2}{*}{ Infants on NIV } & \multirow{2}{*}{$\begin{array}{l}\text { Age [mean } \pm \text { SD } \\
\text { or median (range) } \\
\text { unless otherwise } \\
\text { stated] }\end{array}$} & \multirow[t]{2}{*}{ Interventions } & \multicolumn{2}{|c|}{ Infant NIV outcomes } \\
\hline & & & & & & & Primary & Secondary \\
\hline $\begin{array}{l}\text { Petrone (55), 2007, } \\
\text { Italy }\end{array}$ & $\begin{array}{l}\text { Quantitative: control } \\
\text { before-after }\end{array}$ & $\mathrm{n} / \mathrm{a}$ & $9(7 / 2)$ & $n=9^{d}$ & $\begin{array}{l}7 \text { months } \\
\text { (2-33 months) }\end{array}$ & $\operatorname{BPAP}(n=9)$ & $\begin{array}{l}\text { - Changes in respi- } \\
\text { ratory parameters }\end{array}$ & $\begin{array}{l}\text { - Number of sub- } \\
\text { jects on NIV }\end{array}$ \\
\hline $\begin{array}{l}\text { Vasconcelos (56), } \\
2005 \text {, Portugal }\end{array}$ & $\begin{array}{l}\text { Quantitative: } \\
\text { observational (cohort) }\end{array}$ & 11 years & $22(16 / 6)$ & $n=7^{d}$ & $\begin{array}{l}\text { Overall: } 5.5 \text { years } \\
\text { (6 months to } \\
26 \text { years) } \\
\text { SMA type } 1 \text { group: } \\
13 \text { months ( } 3 \text { months } \\
\text { to } 3 \text { years) }\end{array}$ & $\begin{array}{l}\text { None }(n=5) \\
\operatorname{BPAP}(n=17)\end{array}$ & $\begin{array}{l}\text { - Hospitalizations } \\
\text { - Survival/mortality }\end{array}$ & $\begin{array}{l}\text { - Number of sub- } \\
\text { jects on NIV } \\
\text { - Benefit of } \\
\text { NIV (growth } \\
\text { parameters) }\end{array}$ \\
\hline \multicolumn{9}{|c|}{ Articles on neuromuscular disease: achondroplasia } \\
\hline $\begin{array}{l}\text { Afsharpaiman (57), } \\
\text { 2011, Iran, Australia }\end{array}$ & $\begin{array}{l}\text { Quantitative: } \\
\text { observational (cohort) }\end{array}$ & 15 years & $46(22 / 24)$ & $n=7$ & $\begin{array}{l}\text { Overall: } 3.9 \text { years } \\
\text { Infants: }<2 \text { years } \\
(n=7)\end{array}$ & $\begin{array}{l}\operatorname{CPAP}(n=9) \\
\text { AT }(n=13)\end{array}$ & & $\begin{array}{l}\text { - Number of sub- } \\
\text { jects on } \mathrm{NIV}^{\star}\end{array}$ \\
\hline \multicolumn{9}{|c|}{ Articles on neuromuscular disease: multiple (spinal muscular atrophy type 1 and congenital myopathy) } \\
\hline $\begin{array}{l}\text { Han (58), 2015, } \\
\text { Korea }\end{array}$ & $\begin{array}{l}\text { Quantitative: } \\
\text { observational (cohort) }\end{array}$ & 13.4 years & $57(\mathrm{n} / \mathrm{a})$ & $\mathrm{n} / \mathrm{a}$ & $\begin{array}{l}\text { Overall: } 7.7 \text { months } \\
\text { (2-158 months) } \\
\text { Infants with SMA type } \\
1: 6.6 \text { months (2-26) } \\
\text { CM: } 7.8 \text { months } \\
(3-121)\end{array}$ & $\begin{array}{l}\mathrm{NIV}(n=8) \\
\operatorname{IMV}(n=46)\end{array}$ & - Survival/mortality & $\begin{array}{l}\text { - Number of sub- } \\
\text { jects on NIV } \\
\text { - NIV success/failure }\end{array}$ \\
\hline
\end{tabular}

Articles on neuromuscular disease: myotonic dystrophy

\begin{tabular}{|c|c|c|c|c|c|c|c|}
\hline $\begin{array}{l}\text { Wood (59), 2017, UK, } \\
\text { Germany }\end{array}$ & $\begin{array}{l}\text { Quantitative: } \\
\text { observational } \\
\text { (cross-sectional) }\end{array}$ & 4 years & $610(272 / 338)$ & $n=2$ & $\begin{array}{l}41.1 \text { years ( } 8 \text { months } \\
\text { to } 78 \text { years) }\end{array}$ & $\operatorname{NIV}(n=35)$ & $\begin{array}{l}\text { - Number of sub- } \\
\text { jects on } \mathrm{NIV}^{*}\end{array}$ \\
\hline
\end{tabular}

Articles on central nervous system disease: congenital hypoventilation syndrome

\begin{tabular}{|c|c|c|c|c|c|c|c|c|}
\hline $\begin{array}{l}\text { Garcia Teresa (60), } \\
\text { 2017, Spain }\end{array}$ & $\begin{array}{l}\text { Quantitative: } \\
\text { observational } \\
\text { (cross-sectional) }\end{array}$ & 3.75 years & $38(17 / 21)$ & $n=8^{d}$ & $\begin{array}{l}11.35 \text { ( } 5 \text { months to } \\
28.6 \text { years) }\end{array}$ & $\operatorname{NIV}(n=8)$ & $\begin{array}{l}\text { - Hospitalizations } \\
\text { - Survival/mortality }\end{array}$ & $\begin{array}{l}\text { - Number of sub- } \\
\text { jects on NIV } \\
\text { - NIV failure/success }\end{array}$ \\
\hline $\begin{array}{l}\text { Hartmann (61), 1994, } \\
\text { UK }\end{array}$ & $\begin{array}{l}\text { Quantitative: } \\
\text { observational (case } \\
\text { series) }\end{array}$ & $\mathrm{n} / \mathrm{a}$ & $9(3 / 6)$ & $n=6$ & 22 days to 52 months & $\begin{array}{l}\operatorname{VNEP}(n=9)^{f} \\
\operatorname{CPAP}(n=3)^{9}\end{array}$ & $\begin{array}{l}\text { - Discontinuation } \\
\text { of NIV }\end{array}$ & $\begin{array}{l}\text { - Number of sub- } \\
\text { jects on NIV } \\
\text { - Benefit of } \\
\text { NIV (growth } \\
\text { parameters) } \\
\text { - NIV success/failure } \\
\text { - Quality of life }\end{array}$ \\
\hline
\end{tabular}


TABLE 2 | Continued

First author, year,

Study design

Study duration

Total $n(\mathrm{M} / \mathrm{F})$

Infants on NIV

Interventions

Infant NIV outcomes

country

or median (range)

unless otherwise

stated]

Khayat (62), 2017,
Canada, USA

$$
\text { Quanitative: }
$$

2.7 years

$8(4 / 4)$

Overall: 10.0 years

BPAP $(n=8)$

Primary

Secondary

Canada, USA

observational (control

(8.4-11.6 years)

infants: 1.1 years

- Number of sub-

before-after

66 days to 59 month

$(n=5)$

$\operatorname{VNEP}(n=5)$
$\operatorname{CPAP}(n=1)$

$\operatorname{IMV}(n=2)$

jects on NIV

- NIV modality

Noyes (63), 1999

analysis

$\mathrm{n} / \mathrm{a}$

$7(3 / 4)$

$n=5$

- Discontinuation

- Number of sub-

jects on NIV

- Benefit of

NIV (growth

parameters)

- Quality of life

\begin{tabular}{|c|c|c|c|c|c|c|}
\hline $\begin{array}{l}\text { Ramesh (64), 2008, } \\
\text { UK }\end{array}$ & $\begin{array}{l}\text { Quantitative: } \\
\text { observational } \\
\text { (cross-sectional) }\end{array}$ & $n / a$ & $15(5 / 10)$ & $n=7$ & $\begin{array}{l}\text { Early start: } 8 \text { weeks } \\
\text { (5-26 weeks) } \\
\text { Late start: } 8 \text { years } \\
\text { ( } 1.5-11 \text { years) }\end{array}$ & $\operatorname{NIV}(n=15)$ \\
\hline
\end{tabular}

\begin{tabular}{ll}
\hline Tibballs (65), 2003, & $\begin{array}{l}\text { Quantitative: } \\
\text { observational (case } \\
\text { Australia }\end{array}$ \\
series)
\end{tabular}

$\mathrm{n} / \mathrm{a}$

$4(2 / 2)$

$n=2$

6 weeks to 9 years

$\operatorname{BPAP}(n=4)$

Changes in respi-
ratory parameters

- Number of sub-

jects on NIV

- Benefit of NIV

(extubation)

- Mask

complications

- Number of sub-

jects on NIV

- Benefit of NIV

(extubation)

- Mask

complications

\section{Articles on cardio-respiratory disease: congenital heart disease}

\begin{tabular}{|c|c|c|c|c|c|c|c|c|}
\hline Bunn (66), 2004, UK & $\begin{array}{l}\text { Quantitative: } \\
\text { observational (case } \\
\text { series) }\end{array}$ & $n / a$ & $4(0 / 4)$ & $n=3$ & 5-34 months & $\mathrm{NIV}(n=4)$ & $\begin{array}{l}\text { - Changes in respi- } \\
\text { ratory parameters } \\
\text { - Discontinuation } \\
\text { of NIV }\end{array}$ & $\begin{array}{l}\text { - Number of sub- } \\
\text { jects on NIV }\end{array}$ \\
\hline
\end{tabular}

\section{Articles on multiple underlying disease conditions}

\begin{tabular}{|c|c|c|c|c|c|c|c|}
\hline $\begin{array}{l}\text { Adeleye (67), 2016, } \\
\text { Canada }\end{array}$ & $\begin{array}{l}\text { Quantitative: } \\
\text { observational (cohort) }\end{array}$ & 5 years & $92(54 / 38)$ & $n=49$ & $208.5 \pm 101.2$ days & $\mathrm{NIV}(n=49)$ & $\begin{array}{l}\text { - Number of sub- } \\
\text { jects on NIV } \\
\text { - Adherence to NIV }\end{array}$ \\
\hline $\begin{array}{l}\text { Amaddeo (3), 2016, } \\
\text { France }\end{array}$ & $\begin{array}{l}\text { Quantitative: } \\
\text { observational (cohort) }\end{array}$ & 1 year & $76(39 / 37)$ & $n / a$ & $\begin{array}{l}\text { Overall for patient } \\
\text { groups: acute: } \\
0.3 \text { year }(0.1-13.5) \\
\text { Sub-acute: } 0.6 \text { year } \\
(0.2-18.2) \\
\text { Chronic: } 1.6 \text { years } \\
(0.1-19.5)\end{array}$ & $\begin{array}{l}\operatorname{CPAP}(n=64) \\
\operatorname{BPAP}(n=12)\end{array}$ & $\begin{array}{l}\text { - Number of sub- } \\
\text { jects on NIV } \\
\text { - Predictors of NIV } \\
\text { requirement }\end{array}$ \\
\hline
\end{tabular}


unless otherwise

stated]

\begin{tabular}{|c|c|c|c|c|c|}
\hline $\begin{array}{l}\text { Bertrand (68), 2006, } \\
\text { Chile }\end{array}$ & $\begin{array}{l}\text { Quantitative: } \\
\text { observational (cohort) }\end{array}$ & 10.5 years & $35(18 / 17)$ & $n=9^{d}$ & $\begin{array}{l}12 \text { months ( } 5 \text { months } \\
\text { to } 14 \text { years) }\end{array}$ \\
\hline
\end{tabular}

Primary Secondary

$\operatorname{CPAP}(n=1)$

$\operatorname{BPAP}(n=8)$

- Hospitalizations

- Number of sub-

$\operatorname{MV}(n=26)$

Chatwin (7), 2015, UK Quantitative:

observational (cohort)

18 years

$449(281 / 168)$

$n=59^{\circ}$

Overall: 10 years

$\mathrm{CPAP}(n=57)$

(3-15 years)

CPAP $(n=57)$

- Discontinuation

jects on NIV

Infants: $<1$ year
$(n=59)$

\begin{tabular}{ll}
\hline Fauroux (69), 2005, & Quantitative: \\
France & observational
\end{tabular}

0.5 year

$40(22 / 18)$

$n=16$

(cross-sectional)

- Number of sub-

jects on NIV*

Kherani (70), 2016,

2016, Quantitative:

23 years

$51(30 / 21)$

$n=25$

\begin{tabular}{|c|c|c|c|c|c|c|c|c|}
\hline $\begin{array}{l}\text { Koontz (71), 2003, } \\
\text { USA }\end{array}$ & $\begin{array}{l}\text { Quantitative: } \\
\text { observational (cohort) }\end{array}$ & $\mathrm{n} / \mathrm{a}$ & $20(\mathrm{n} / \mathrm{a})$ & $n=6$ & $1-2$ years & $\operatorname{BPAP}(n=6)$ & & $\begin{array}{l}\text { - Number of sub- } \\
\text { jects on NIV } \\
\text { - Adherence to NIV }\end{array}$ \\
\hline $\begin{array}{l}\text { Machaalani (72), } \\
\text { 2016, Australia }\end{array}$ & $\begin{array}{l}\text { Quantitative: } \\
\text { observational (cohort) }\end{array}$ & 2 years & $99(63 / 36)$ & $n=22$ & $\mathrm{n} / \mathrm{a}$ & $\begin{array}{l}\operatorname{CPAP}(n=55) \\
\operatorname{BPAP}(n=44)\end{array}$ & & $\begin{array}{l}\text { - Number of sub- } \\
\text { jects on NIV* }\end{array}$ \\
\hline $\begin{array}{l}\text { Markstrom (9), 2008, } \\
\text { Sweden }\end{array}$ & $\begin{array}{l}\text { Quantitative: } \\
\text { observational (cohort) }\end{array}$ & 7 years & $18(11 / 7)$ & $n=18$ & $\begin{array}{l}4 \text { months } \\
\text { (1-12 months) }\end{array}$ & $\operatorname{BPAP}(n=18)$ & $\begin{array}{l}\text { - Changes in respi- } \\
\text { ratory parameters } \\
\text { - Discontinuation } \\
\text { of NIV }\end{array}$ & $\begin{array}{l}\text { - Number of sub- } \\
\text { jects on NIV }\end{array}$ \\
\hline $\begin{array}{l}\text { Nathan (73), 2017, } \\
\text { Malaysia }\end{array}$ & $\begin{array}{l}\text { Quantitative: } \\
\text { observational (cohort) }\end{array}$ & 13 years & $70(40 / 30)$ & $n=51$ & $\begin{array}{l}\text { Overall: } 12 \text { months } \\
\text { CPAP: } 6 \text { months } \\
\text { (3-12 months) } \\
\text { BPAP: } 12 \text { months } \\
\text { (5-33 months) } \\
\text { IMV: } 30 \\
\text { (12-57 months) }\end{array}$ & $\begin{array}{l}\operatorname{CPAP}(n=30) \\
\operatorname{BPAP}(n=30) \\
\operatorname{IMV}(n=10)\end{array}$ & $\begin{array}{l}\text { - } \text { Discontinuation } \\
\text { of NIV } \\
\text { - Hospitalizations } \\
\text { - Survival/mortality }\end{array}$ & $\begin{array}{l}\text { - Number of sub- } \\
\text { jects on NIV } \\
\text { - Predictors of NIV } \\
\text { - NIV modality }\end{array}$ \\
\hline $\begin{array}{l}\text { Ramirez (74), 2012, } \\
\text { France }\end{array}$ & $\begin{array}{l}\text { Quantitative: } \\
\text { observational (case } \\
\text { series) }\end{array}$ & 18 months & $97(\mathrm{n} / \mathrm{a})$ & $n=18$ & $\begin{array}{l}\text { Infants: }<2 \text { years } \\
(n=18)\end{array}$ & CPAP and BPAP (n/a) & & $\begin{array}{l}\text { - Number of sub- } \\
\text { jects on NIV* }\end{array}$ \\
\hline
\end{tabular}

Overall: 10.0 years

(0.6-18 years)

Infant: 1.8 years

$\operatorname{NIV}(n=40)$

(0.2-15.3 years)

NIPPV: 0.6 year

(0.4-0.7 year)

IMV: 0.4 year

(0.1-0.7 year)

$\operatorname{IMV}(n=26)$

Changes in respi-
ratory parameters

- Discontinuation

of NIV

- Survival/mortality

- Number of sub

jects on NIV

- Adherence to NIV

Number of sub-

jects on NIV

umber of sub-

Number of sub-

Changes in respi-

Number of sub-

Discontinuation

Discontinuation

- Number of sub-

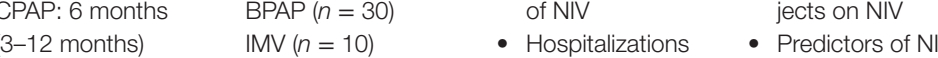

BPAP: 12 month

Survival/mortality 
TABLE 2 | Continued

\begin{tabular}{|c|c|c|c|c|c|c|c|c|}
\hline \multirow{2}{*}{$\begin{array}{l}\text { First author, year, } \\
\text { country }\end{array}$} & \multirow[t]{2}{*}{ Study design } & \multirow[t]{2}{*}{ Study duration } & \multirow[t]{2}{*}{ Total $n(\mathrm{M} / \mathrm{F})$} & \multirow[t]{2}{*}{ Infants on NIV } & \multirow{2}{*}{$\begin{array}{l}\text { Age [mean } \pm \text { SD } \\
\text { or median (range) } \\
\text { unless otherwise } \\
\text { stated] }\end{array}$} & \multirow[t]{2}{*}{ Interventions } & \multicolumn{2}{|c|}{ Infant NIV outcomes } \\
\hline & & & & & & & Primary & Secondary \\
\hline $\begin{array}{l}\text { Zhou (75), 2012, } \\
\text { China }\end{array}$ & $\begin{array}{l}\text { Quantitative: } \\
\text { observational (cohort) }\end{array}$ & 2 years & $14(12 / 2)$ & $n=6^{c}$ & $\begin{array}{l}\text { Overall: } 50 \text { days to } \\
12 \text { years } \\
\text { Infants: }<1 \text { year } \\
(n=6)\end{array}$ & $\begin{array}{l}\operatorname{CPAP}(n=1) \\
\operatorname{BPAP}(n=13)\end{array}$ & & $\begin{array}{l}\text { - Number of sub- } \\
\text { jects on } \mathrm{NIV}^{*}\end{array}$ \\
\hline
\end{tabular}

Studies have been classified according to the primary disease category and disease condition reported. Studies with multiple disease categories have been included at the end of the table.

AT, adenotonsillectomy; CPAP, continuous positive airway pressure; BPAP, bi-level positive airway pressure; IMV, invasive mechanical ventilation; n/a, data not available/reported; MDO, mandibular distraction osteogenesis; NIV, noninvasive ventilation; NPA, nasopharyngeal airway; SMA, spinal muscular atrophy; VNEP, negative extra-thoracic pressure ventilation.

${ }^{*}$ Articles reporting only on the number of subjects using NIV were excluded from synthesis.

${ }^{a}$ Four patients did not tolerate CPAP.

${ }^{b}$ Full list of non-surgical and surgical interventions are in the full text of article.

Number of patients less than 1 year of age.

${ }^{d}$ Determined by the mean/median age of the population during NIV initiation.

${ }^{e}$ Determined by age at first respiratory decompensation.

VNEP failed in two patients.

${ }^{9}$ CPAP used in conjunction with VNEP.

${ }^{h}$ Compared intelligent volume-assured pressured support BPAP to traditional BPAP.

'Only includes infants in the obstructive sleep apnea group.

TABLE 3 | Studies on infants using long-term NIV reporting change in respiratory parameters and discontinuation outcomes

\begin{tabular}{|c|c|c|c|c|c|c|c|c|c|c|c|c|}
\hline \multirow[t]{2}{*}{$\begin{array}{l}\text { First author, } \\
\text { year, country }\end{array}$} & \multirow[t]{2}{*}{ Study design } & \multirow[t]{2}{*}{$\begin{array}{l}\text { Primary } \\
\text { diagnosis }\end{array}$} & \multirow[t]{2}{*}{$\begin{array}{l}\text { Infants using } \\
\text { NIV }\end{array}$} & \multirow[t]{2}{*}{$\begin{array}{l}\text { Age } \\
\text { mean } \pm \text { SD or } \\
\text { med (range) }\end{array}$} & \multirow[t]{2}{*}{ NIV type } & \multicolumn{2}{|l|}{$\begin{array}{l}\text { Total apneas } \\
\text { (mean } \pm \text { SD } \\
\text { events/hour) }\end{array}$} & \multirow{2}{*}{$\begin{array}{c}\begin{array}{c}\text { Obstructive } \\
\text { apneas } \\
\text { (mean } \pm \text { SD } \\
\text { events/hour) }\end{array} \\
\text { Pre-NIV }\end{array}$} & \multicolumn{3}{|c|}{$\begin{array}{c}\begin{array}{c}\text { Central } \\
\text { apneas }\end{array} \\
\text { (mean } \pm \text { SD } \\
\text { events/hour) }\end{array}$} & \multirow[t]{2}{*}{$\begin{array}{l}\text { Infants who } \\
\text { discontinued } \\
(\%)\end{array}$} \\
\hline & & & & & & Pre-NIV & Post-NIV & & Post-NIV & Pre-NIV & Post-NIV & \\
\hline $\begin{array}{l}\text { Harrington } \\
\text { (22), 2003, } \\
\text { Australia, } \\
\text { Finland }\end{array}$ & $\begin{array}{l}\text { P, Obs: } \\
\text { case-control }\end{array}$ & OSA & $n=6$ & $13 \pm 4$ weeks & CPAP & & & $17 \pm 6$ & $1 \pm 1^{*}$ & & & \\
\hline $\begin{array}{l}\text { Downey (20), } \\
\text { 2000, USA }\end{array}$ & $\begin{array}{l}\text { R, Obs: } \\
\text { cohort }\end{array}$ & OSA & $n=18$ & $<2$ years & CPAP & $12.8 \pm 20.0$ & $4.5 \pm 13.4^{\dagger}$ & $4.7 \pm 13.4$ & $2.0 \pm 7.3^{\dagger}$ & & & 90 \\
\hline $\begin{array}{l}\text { McNamara } \\
\text { (27), 1995, } \\
\text { Australia }\end{array}$ & $\begin{array}{l}\text { P, control } \\
\text { before-after }\end{array}$ & OSA & $n=5$ & 8-12 weeks & CPAP & $\begin{array}{r}{ }^{a} 65.6 \pm 14.6 \\
b 106.1 \pm 13.9\end{array}$ & $\begin{array}{l}10.5 \pm 14.6^{\star \star} \\
26.6 \pm 13.9^{\star *}\end{array}$ & $\begin{array}{l}\text { a29.3 } \pm 9.4 \\
80.8 \pm 16.8\end{array}$ & $\begin{array}{l}\text { a } 0.3 \pm 9.4^{\star *} \\
\text { b2.0 } \pm 16.8^{* \star}\end{array}$ & $\begin{array}{l}36.5 \pm 6.6 \\
25.6 \pm 4.5\end{array}$ & $\begin{array}{l}10.3 \pm 6.6^{* *} \\
24.6 \pm 4.5\end{array}$ & 100 \\
\hline $\begin{array}{l}\text { McNamara } \\
\text { (28), 1999, } \\
\text { Australia }\end{array}$ & P, Obs: cohort & OSA & $n=24$ & $1-51$ weeks & CPAP & $\begin{array}{l}44.4 \pm 9.3 \\
68.6 \pm 8.9\end{array}$ & $\begin{array}{r}9.5 \pm 1.2^{*} \\
22.7 \pm 2.3^{*}\end{array}$ & $\begin{array}{l}14.6 \pm 3.9 \\
43.6 \pm 8.3\end{array}$ & $\begin{array}{l}0.1 \pm 0.1^{*} \\
0.4 \pm 0.1^{*}\end{array}$ & $\begin{array}{l}29.8 \pm 7.6 \\
25.0 \pm 4.3\end{array}$ & $\begin{array}{c}9.4 \pm 1.2^{*} \\
22.3 \pm 2.2\end{array}$ & 72 \\
\hline
\end{tabular}


TABLE 3 | Continued

\begin{tabular}{|c|c|c|c|c|c|c|c|c|c|c|c|}
\hline $\begin{array}{l}\text { McNamara } \\
\text { (29), 1999, } \\
\text { Australia }\end{array}$ & $\begin{array}{l}\text { P, Obs: } \\
\text { case-control }\end{array}$ & OSA & $n=8$ & $\begin{array}{l}10.8 \pm 1.3 \\
\text { weeks }\end{array}$ & CPAP & & $\begin{array}{l}22.2 \pm 8.8 \\
54.8 \pm 16.3\end{array}$ & $\begin{array}{l}10.6 \pm 2.6^{\star} \\
25.7 \pm 7.2^{\star}\end{array}$ & $\begin{array}{l}36.1 \pm 8.6 \\
32.9 \pm 8.1\end{array}$ & $\begin{array}{l}26.3 \pm 7.4 \\
38.2 \pm 8.2\end{array}$ & \\
\hline $\begin{array}{l}\text { Leonardis } \\
\text { (23), 2013, } \\
\text { USA }\end{array}$ & $\begin{array}{l}\text { R, Obs: } \\
\text { cohort }\end{array}$ & OSA & $n=18$ & $16.0 \mathrm{mo}$ & $\begin{array}{l}\text { CPAP } \\
\text { BPAP }\end{array}$ & $\begin{array}{l}\% \text { decrease in } \\
\mathrm{AHI}: 67.2^{*}\end{array}$ & & & & & \\
\hline $\begin{array}{l}\text { Robison (10), } \\
\text { 2013, USA }\end{array}$ & $\begin{array}{l}\text { R, Obs: } \\
\text { cohort }\end{array}$ & OSA & $n=18$ & $\begin{array}{l}15.6 \text { months } \\
\text { (3-29 months) }\end{array}$ & $\begin{array}{l}\text { CPAP } \\
\text { BPAP }\end{array}$ & $\begin{array}{l}\% \text { decrease in } \\
\mathrm{AHI}: 84.1^{*}\end{array}$ & & & & & \\
\hline $\begin{array}{l}\text { Guilleminault } \\
\text { (21), 1995, } \\
\text { USA }\end{array}$ & $\begin{array}{l}\text { P, Obs: } \\
\text { case-control }\end{array}$ & OSA & $n=72$ & $\begin{array}{l}24 \pm 9 \text { weeks } \\
\text { (4-43 months) }\end{array}$ & CPAP & & & & & & 14 \\
\hline $\begin{array}{l}\text { Rosen (30), } \\
\text { 2010, United } \\
\text { States }\end{array}$ & $\begin{array}{l}\text { R, Obs: } \\
\text { cohort }\end{array}$ & OSA & $n=6$ & $<2$ years & CPAP & & & & & & 50 \\
\hline \multirow{2}{*}{$\begin{array}{l}\text { First author, } \\
\text { year, country }\end{array}$} & Study design & Primary & Infants using & & NIV type & \multicolumn{5}{|c|}{ Change in respiratory parameters } & Infants who \\
\hline & & Diagnosis & & med (range) & & \multicolumn{3}{|c|}{ Variables } & $\begin{array}{c}\text { Pre-NIV, } \\
\text { mean } \pm \text { SD }\end{array}$ & $\begin{array}{c}\text { Post-NIV, } \\
\text { mean } \pm \text { SD }\end{array}$ & (\%) \\
\hline $\begin{array}{l}\text { Leboulanger } \\
\text { (36), 2010, } \\
\text { France }\end{array}$ & $\begin{array}{l}\text { P; Obs: case } \\
\text { series }\end{array}$ & PRS & $n=7$ & $\begin{array}{l}2 \text { months } \\
\text { (1-10 months) }\end{array}$ & $\begin{array}{l}\operatorname{CPAP}(n=5) \\
\operatorname{BPAP}(n=2)\end{array}$ & \multicolumn{3}{|c|}{$\begin{array}{l}\text { RR (breaths/minute) } \\
T_{1} / T_{\text {TOT }}(\%) \\
P_{\text {es }} \text { swing }\left(\mathrm{cm} \mathrm{H}_{2} \mathrm{O}\right) \\
P_{\text {di swing }}\left(\mathrm{cm} \mathrm{H}_{2} \mathrm{O}\right) \\
\text { Total sleep time with } \mathrm{S}_{\mathrm{p}} \mathrm{O}_{2}<90 \%(\%) \\
\text { Total sleep time with } \mathrm{P}_{\mathrm{a}} \mathrm{CO}_{2}>50 \mathrm{~mm} \mathrm{Hg}(\%)\end{array}$} & $\begin{array}{l}55 \pm 9 \\
59 \pm 9 \\
29 \pm 13 \\
31 \pm 12 \\
14 \pm 10 \\
88 \pm 12\end{array}$ & $\begin{aligned} 37 & \pm 7 \\
40 & \pm 7^{\star} \\
9 & \pm 4^{*} \\
12 & \pm 5^{\star} \\
1 & \pm 2^{*} \\
0 & \pm 0^{+}\end{aligned}$ & 71 \\
\hline $\begin{array}{l}\text { Amaddeo } \\
\text { (31), 2016, } \\
\text { France }\end{array}$ & $\begin{array}{l}\text { R; Obs: } \\
\text { cohort }\end{array}$ & PRS & $n=9$ & $0-2$ months & CPAP & \multicolumn{3}{|c|}{$\begin{array}{l}\text { Apnea-hypopnea index (events/hour) } \\
\text { Oxygen desaturation index (events/hour) } \\
\text { Minimum } \mathrm{S}_{\mathrm{p}} \mathrm{O}_{2}(\%) \\
\% \text { time } \mathrm{S}_{\mathrm{p}} \mathrm{O}_{2}<90 \% \\
\text { Maximum } \mathrm{P}_{\mathrm{a}} \mathrm{CO}_{2}(\%)\end{array}$} & $\begin{array}{l}19-42 \\
18-137 \\
78-90 \\
0-16 \\
41-55\end{array}$ & $\begin{array}{l}\text { Normal PG } \\
\text { and/or gas } \\
\text { exchange } \\
\text { (reported } \\
\text { narratively) }\end{array}$ & 66 \\
\hline $\begin{array}{l}\text { First author, } \\
\text { year, country }\end{array}$ & Study design & $\begin{array}{l}\text { Primary } \\
\text { diagnosis }\end{array}$ & $\begin{array}{c}\text { Infants using } \\
\text { NIV }\end{array}$ & $\begin{array}{l}\text { Age, } \\
\text { mean } \pm \text { SD or } \\
\text { med (range) }\end{array}$ & NIV type & Variables & \multicolumn{2}{|c|}{ Supportive care } & CPAP & BPAP & $\begin{array}{l}\text { Infants who } \\
\text { discontinued } \\
(\%)\end{array}$ \\
\hline $\begin{array}{l}\text { Essouri (38), } \\
\text { 2005, France }\end{array}$ & $\begin{array}{l}\text { P; Obs: } \\
\text { case-control }\end{array}$ & LTM & $n=10$ & $\begin{array}{l}9.5 \text { months } \\
\text { ( } 3-18 \text { months) }\end{array}$ & $\begin{array}{l}\text { CPAP } \\
(n=10) \\
\text { BPAP } \\
(n=10)\end{array}$ & $\begin{array}{l}\text { RR (breaths/minute) } T_{1} / T_{\text {TOT }}(\%) \\
P_{\text {es }} \text { swing }\left(\mathrm{cm} \mathrm{H}_{2} \mathrm{O}\right) \\
P_{\text {di }} \text { swing }\left(\mathrm{cm} \mathrm{H}_{2} \mathrm{O}\right) \\
\text { PTP }_{\text {es }} \text { minute }\left(\mathrm{cm} \mathrm{H}_{2} \mathrm{O} / \text { second/ }\right. \\
\text { minute) } \\
\text { PTP }_{\text {ditminute }\left(\mathrm{cm} \mathrm{H}_{2} \mathrm{O} / \text { second/ }\right.} \\
\text { minute) }\end{array}$ & \multicolumn{2}{|c|}{$\begin{array}{c}45(24-84) \\
63(35-86) \\
28(13-76) \\
30(16-75) \\
695(364-1417) \\
845(159-1183)\end{array}$} & $\begin{array}{c}29(18-60) \\
41(34-60)^{\star \star} \\
10(7-28)^{\star \star} \\
12(8-32)^{\star \star} \\
143 \\
(98-469)^{\star \star} \\
195 \\
(115-434)^{\star \star}\end{array}$ & $\begin{array}{c}25(14-50)^{* c} \\
48(28-55)^{\star \star} \\
13(6-33)^{\star \star} \\
14(7-33)^{\star \star} \\
211 \\
(73-588)^{\star \star} \\
248 \\
(45-784)^{\star \star}\end{array}$ & \\
\hline
\end{tabular}


TABLE 3 | Continued

\begin{tabular}{|c|c|c|c|c|c|c|c|c|c|}
\hline $\begin{array}{l}\text { Fauroux (39), } \\
\text { 2001, France, } \\
\text { UK }\end{array}$ & $\begin{array}{l}\text { P; Obs: } \\
\text { case-control }\end{array}$ & LTM & $n=5$ & $8-19$ months & CPAP & $\begin{array}{l}\mathrm{S}_{\mathrm{p}} \mathrm{O}_{2}(\%) \\
\mathrm{S}_{\mathrm{p}} \mathrm{O}_{2} \text { nadir (\%) } \\
\% \text { sleep with } \mathrm{S}_{\mathrm{p}} \mathrm{O}_{2}<90 \%\end{array}$ & $\begin{array}{l}91.7 \pm 2.3 \\
74.7 \pm 7.5 \\
29.5 \pm 19.6\end{array}$ & $\begin{aligned} 96.2 & \pm 2.0^{*} \\
88.0 & \pm 2.5^{*} \\
0.5 & \pm 0.8^{*}\end{aligned}$ & 60 \\
\hline $\begin{array}{l}\text { Zwacka } \\
\text { (41), 1997, } \\
\text { Germany }\end{array}$ & $\begin{array}{l}\text { R: Obs: } \\
\text { cohort }\end{array}$ & LTM & $n=7$ & $\begin{array}{l}3 \text { weeks to } 3 \\
\text { months }\end{array}$ & CPAP & $\begin{array}{l}\mathrm{HR} \text { (beats/minute) } \\
\mathrm{RR} \text { (breaths/minute) } \\
\mathrm{S}_{\mathrm{a}} \mathrm{O}_{2} \text { in REM sleep (\%) } \\
\mathrm{S}_{\mathrm{a}} \mathrm{O}_{2} \text { in NREM sleep (\%) }\end{array}$ & $\begin{array}{c}135-160 \\
34-42 \\
60-95 \\
85-98\end{array}$ & $\begin{array}{c}110-130 \\
22-28 \\
88-100 \\
92-100\end{array}$ & \\
\hline $\begin{array}{l}\text { Shatz (40), } \\
\text { 2004, Israel }\end{array}$ & $\begin{array}{l}\text { R; Obs: } \\
\text { cohort }\end{array}$ & LTM & $n=14$ & $\begin{array}{l}6.5 \pm 3.5 \\
\text { months } \\
\text { (1-18 months) }\end{array}$ & $\begin{array}{l}\operatorname{CPAP}(n=5) \\
\operatorname{BPAP}(n=9)\end{array}$ & & & & 100 \\
\hline $\begin{array}{l}\text { First author, } \\
\text { year, country }\end{array}$ & Study design & $\begin{array}{l}\text { Primary } \\
\text { diagnosis }\end{array}$ & $\begin{array}{l}\text { Infants using } \\
\text { NIV }\end{array}$ & $\begin{array}{l}\text { Age, } \\
\text { mean } \pm \text { SD or } \\
\text { med (range) }\end{array}$ & NIV type & Change in respiratory pa & & & $\begin{array}{l}\text { Infants who } \\
\text { discontinued } \\
\text { (\%) }\end{array}$ \\
\hline $\begin{array}{l}\text { Tibballs } \\
\text { (65), 2003, } \\
\text { Australia }\end{array}$ & $\begin{array}{l}\text { R; Obs: case } \\
\text { series }\end{array}$ & $\mathrm{CHS}$ & $n=2$ & $\begin{array}{l}6 \text { weeks and } \\
9 \text { months }\end{array}$ & $\begin{array}{l}\operatorname{BPAP}(n=2) \\
\operatorname{VNEP}(n=2)\end{array}$ & \multicolumn{4}{|c|}{ Decrease in $\mathrm{PaCO}_{2}$ to $40-50 \mathrm{~mm} \mathrm{Hg}$ in one infant } \\
\hline $\begin{array}{l}\text { Hartmann } \\
\text { (61), 1994, U }\end{array}$ & $\begin{array}{l}\text { P; Obs: case } \\
\text { series }\end{array}$ & $\mathrm{CHS}$ & $n=6$ & $\begin{array}{l}22 \text { days to } \\
5 \text { months }\end{array}$ & $\begin{array}{l}\operatorname{VNEP}(n=6) \\
\operatorname{CPAP}(n=2)\end{array}$ & \multicolumn{3}{|c|}{ Improvements in hypoventilation in three patients (reported narratively) } & 33 \\
\hline $\begin{array}{l}\text { Noyes (63), } \\
\text { 1999, UK }\end{array}$ & $\begin{array}{l}\text { P; Obs: } \\
\text { cross-sectional }\end{array}$ & $\mathrm{CHS}$ & $n=5$ & $\begin{array}{l}66 \text { days to } \\
59 \text { months }\end{array}$ & $\begin{array}{l}\operatorname{VNEP}(n=5) \\
\operatorname{CPAP}(n=1)\end{array}$ & & & & 33 \\
\hline $\begin{array}{l}\text { Ramesh (64), } \\
\text { 2008, UK }\end{array}$ & $\begin{array}{l}\text { P; Obs: } \\
\text { cross-section }\end{array}$ & $\mathrm{CHS}$ & $n=6$ & $\begin{array}{l}8 \text { weeks } \\
\text { (5-26 weeks) }\end{array}$ & & & & & 0 \\
\hline
\end{tabular}

AHI, apnea-hypopnea index (events/hour); BPAP, bi-level positive airway pressure; CHS, congenital hypoventilation syndrome; CPAP, continuous positive airway pressure; HR, heart rate; LTM, laryngo-tracheomalacia; NIV, non-invasive ventilation; Obs, observational study; OSA, obstructive sleep apnea; $P$, prospective; $P_{a} C_{2}$, partial pressure of carbon dioxide; $P_{\text {dit }}$ diaphragmatic pressure; $P_{\text {es, }}$ esophageal pressure; $P G$, polygraphy; PRS, Pierre Robin sequence; $R$, retrospective; $R R$, respiratory rate; $\mathrm{S}_{2} \mathrm{O}_{2}$, oxygen saturation; $\mathrm{SPO}_{2}$, pulse oximetry; $T_{J} T_{\text {Tot, }}$ inspiratory time/total respiratory cycle time; VNEP, negative extra-thoracic pressure ventilation.

${ }^{*} p<0.05$.

${ }^{* *} p<0.01$

$+p<0.001$.

${ }^{2}$ Apneas seen in non-rapid eye movement (NREM) sleep.

${ }^{\mathrm{D}} \mathrm{Apneas}$ seen in rapid eye movement (REM) sleep. 
also reported an average NIV use per day of 10.2 hours/day in seven infants (50).

\section{Spinal Muscular Atrophy Type 1}

There were 14 studies $(14 / 60,23 \%)$ of infants with spinal muscular atrophy type 1 (SMA1) using long-term NIV (Table 2). Twelve of these studies reported on primary or secondary outcomes and were synthesized (43-46, 48-51, 53-56). Only one study (1/12, $8 \%$ ) reported on changes in respiratory parameters and showed improvements in respiratory effort and normalization of respiratory gases in SMA1 patients using NIV therapy (Table 3) (55). Six studies $(6 / 12,50 \%)$ reported on hospitalization outcomes (Table 4) $(43,44,46,51,53,54)$. Of these, two studies reported that hospitalizations per patient per year were significantly higher in infants on NIV than infants with a tracheostomy until after three years of age $(44,46)$. Nine studies $(9 / 12,75 \%)$ reported on mortality outcomes $(43,44,46,48,49,51,53,54,56)$; four of these studies compared infants on supportive care with those using NIV, showing prolonged survival in the NIV group (44, $46,51,53)$. Three studies $(3 / 12,25 \%)$ reported improvements in growth parameters, seen by resolution of chest wall deformity (pectus excavatum) after the initiation of NIV therapy $(43,45$, 49). An additional three studies showed that NIV helped facilitate extubation in infants with SMA1 $(43,48,50)$.

\section{Central Hypoventilation Syndrome}

There were six studies $(6 / 60,10 \%)$ on NIV use for infants with central hypoventilation syndrome (CHS) that reported primary or secondary outcomes, and all six were summarized (Table 2) (60-65). The diagnosis of CHS was confirmed clinically in two studies $(61,65)$, via PHOX2B gene mutation analysis in three studies $(60,62,64)$, and unreported in one study (63). NIV was used in conjunction with negative extra-thoracic pressure ventilation (VNEP) therapy in two studies: in one study, it was used as the primary therapy (65) and, in the second study, CPAP was used to relieve upper airway obstruction not resolved with VNEP (61). Improvements in respiratory parameters were reported in two studies: one showed the normalization of the partial pressure of carbon dioxide and resolution of pulmonary hypertension following the use of NIV (65) and the other study showed improvements in hypoventilation for 50\% (3/6) of infants (Table 3) (61). One study with six infants reported NIV discontinuation in two infants (33\%) because of improvements in respiratory parameters; the remaining four infants were using NIV only during sleep (61). One cohort study reported mortality outcomes and a higher hospitalization time for infants using invasive mechanical ventilation compared to NIV (Table 4) (60). Two studies showed parent-reported improvements in growth and development after NIV initiation using the results of a parent questionnaire (61, 63). An additional two studies reported pressure-related effects of mask use, which were predominantly skin breakdown and mid-face hypoplasia $(64,65)$. One cross-sectional study showed that it took less than a week for five of the six infants to adjust to NIV (61). A control before-after study of infants using two BPAP ventilators showed comparable sleep and respiratory parameters with both ventilators, with the exception of a greater decrease in the maximum transcutaneous carbon dioxide with the intelligent volume-assured pressured support compared to a traditional BPAP ventilator (62).

\section{Synthesized Findings}

After examining studies for all disease categories and respective outcomes, only three studies on infants with SMA1 reporting mortality outcomes were eligible for meta-analysis $(44,46,51)$. The results of meta-analysis showed that there was a statistically significant decrease in the relative risk of mortality in the NIV group compared to the supportive care group (Figure 2).

\section{Risk of Bias and Quality Assessment of Outcomes}

Risk of bias ranged from moderate to severe in all studies synthesized in this review (Table 5). Study design was the main contributor to the low quality assessment of the studies. Almost all the included studies had an observational study design, which contributed to confounding bias in participant selection and selected reporting of results. Grading of the quality of the evidence for outcomes such as changes in respiratory parameters, discontinuation of NIV, hospitalizations, and mortality showed that the quality of evidence ranged from low to very low for all studies (Table 6).

\section{DISCUSSION}

\section{Summary of Main Findings}

To our knowledge, this is the first systematic review on the use of long-term NIV in infants. We identified studies on a diverse range of airway conditions in which NIV therapy improved the results of polysomnographic and respiratory parameters. With data available for NMD and CNS disorders limited to SMA1 and CHS, extrapolation of NIV benefits to other NMD and CNS disorders in infants is challenging. Not all outcomes were studied in all disease categories; length of hospitalization was the focus in studies of PRS, while hospitalizations and mortality were the focus in studies of SMA, and respiratory events and NIV discontinuation in the remaining groups. The overall quality of evidence to support appropriate conclusions was low to very low for all studies included in this review.

There is a diverse range of airway disorders that may benefit from NIV therapy. Previous studies have identified many conditions that can predispose infants to upper airway obstruction, including craniofacial disorders, laryngeal disorders, and nasal obstruction (76). Similarly, in this review, we identified NIV use in a wide variety of diseases associated with compromised airway function, the most common being OSA, PRS, ALTE, infants at risk for SIDS, and LTM. The improvement in respiratory parameters reported in infants with airway disorders reflects an overall benefit from NIV therapy. In addition, the underlying airway conditions have potential for improvement, as seen with the infants discontinuing due to underlying improvements, so there may be less risk with NIV compared to invasive mechanical ventilation. Extrapolating these results to conditions with a similar pathophysiology, but for which there is no evidence for NIV use in the literature, may be reasonable given the diversity of disorders represented in the available evidence. 
TABLE 4 | Studies on infants using long-term NIV reporting hospitalization and mortality outcomes.

First author, Study design Primary Infants using Age, mean \pm SD NIV typ

year, country

diagnosis

NIV

or med (range)

Hospitalization (per infant/year unless otherwise

Mortality ( $\%$ of total infants unless

stated)

otherwise stated)

\begin{tabular}{lllllll}
\hline Supportive & NIV & IMV & Supportive & NIV & IMV
\end{tabular}

care

care

\begin{tabular}{|c|c|c|c|c|c|c|c|c|c|c|}
\hline $\begin{array}{l}\text { Bach (46), } \\
\text { 2007, USA }\end{array}$ & R; Obs: cohort & SMA1 & $n=47$ & $10.6 \pm 5.7$ months & BPAP & 1.58 & $0.37^{\dagger}$ & 100 & 17 & 19 \\
\hline $\begin{array}{l}\text { Bach (44), } \\
\text { 2002, USA }\end{array}$ & $\begin{array}{l}\text { R; Obs: case } \\
\text { series }\end{array}$ & SMA1 & $n=33$ & $11.2 \pm 5.7$ months & BPAP & 1.53 & $0.58^{\star}$ & 100 & 6 & 6 \\
\hline Gregoretti (51), & R; Obs: case & SMA1 & $n=31$ & $12.6 \pm 14.4$ months & BPAP & 0.023 & 0.006 & 93 & 45 & 17 \\
\hline
\end{tabular}
series

n=16 $10.4 \pm 6.2$ months

0.15

13

2011, Italy

Bach (43), R; Obs: case $\quad$ SMA1 $\quad n=8 \quad 3-28$ months

2000, USA series:

Birnkrant (48), R; Obs: case SMA1 $\quad n=3 \quad 4-9$ months $\quad$ BPAP

1998, USA

Chatwin (49), R; Obs: cohort SMA

2011, UK

Vasconcelos R; Obs: cohort SMA

(56), 2005,

Portugal

$n=3 \quad 4-9$ months

BPAP

00

Lemoine (53), R; Obs: cohort SMA1

2012, USA

A1

$n=13 \quad 11$ months $\quad$ BPAP

11 months

BPAP

38

$=7 \quad 13$ months $\quad$ BPAP

13 months

3 years)

(n)

$=26 \quad 136$ days $(54-196) \quad$ BPAP

P

$46 \% \quad 83 \%$


TABLE 4 | Continued

\begin{tabular}{|c|c|c|c|c|c|c|c|c|c|c|c|}
\hline \multirow[t]{2}{*}{$\begin{array}{l}\text { First author, } \\
\text { year, country }\end{array}$} & \multirow[t]{2}{*}{ Study design } & \multirow[t]{2}{*}{$\begin{array}{l}\text { Primary } \\
\text { diagnosis }\end{array}$} & \multirow[t]{2}{*}{$\begin{array}{l}\text { Infants using } \\
\text { NIV }\end{array}$} & \multirow[t]{2}{*}{$\begin{array}{l}\text { Age, mean } \pm \text { SD } \\
\text { or med (range) }\end{array}$} & \multirow[t]{2}{*}{ NIV type } & \multicolumn{3}{|c|}{$\begin{array}{l}\text { Hospitalization (per infant/year unless otherwise } \\
\text { stated) }\end{array}$} & \multicolumn{3}{|c|}{$\begin{array}{l}\text { Mortality (\% of total infants unless } \\
\text { otherwise stated) }\end{array}$} \\
\hline & & & & & & $\begin{array}{l}\text { Supportive } \\
\text { care }\end{array}$ & NIV & IMV & $\begin{array}{l}\text { Supportive } \\
\text { care }\end{array}$ & NIV & IMV \\
\hline $\begin{array}{l}\text { Amaddeo (31), } \\
\text { 2016, France }\end{array}$ & R; Obs: cohort & PRS & $n=9$ & 0-2 months & CPAP & & $\begin{array}{c}1 \text { month } \\
\text { (20-40 days) }\end{array}$ & $\begin{array}{c}2 \text { months } \\
\text { (6 weeks to } \\
4 \text { months) }\end{array}$ & & & \\
\hline $\begin{array}{l}\text { Kam (35), } \\
\text { 2015, Canada }\end{array}$ & R; Obs: cohort & PRS & $n=20$ & $\begin{array}{l}23 \text { months ( } 5 \text { days } \\
\text { to } 8 \text { years) }\end{array}$ & CPAP & $28 \pm 24$ days & $66 \pm 46$ days & $138 \pm 76$ days $^{\dagger}$ & NR & & \\
\hline $\begin{array}{l}\text { First author, } \\
\text { year, country }\end{array}$ & Study design & $\begin{array}{l}\text { Primary } \\
\text { diagnosis }\end{array}$ & $\begin{array}{l}\text { Infants using } \\
\text { NIV }\end{array}$ & $\begin{array}{l}\text { Age, mean } \pm \text { SD } \\
\text { or med (range) }\end{array}$ & NIV type & Hospitalization & per infant/year & r $\%$ of total) & $\begin{array}{c}\text { Mortality } \\
\text { (\% of total } \\
\text { infants) }\end{array}$ & & \\
\hline $\begin{array}{l}\text { McNamara } \\
\text { (27), 1995, } \\
\text { Australia }\end{array}$ & P, Obs: cohort & OSA & $n=5$ & 8-12 weeks & CPAP & - & & & $0 \%$ & & \\
\hline \multirow[t]{2}{*}{$\begin{array}{l}\text { First author, } \\
\text { year, country }\end{array}$} & Study design & $\begin{array}{l}\text { Primary } \\
\text { diagnosis }\end{array}$ & $\begin{array}{l}\text { Infants using } \\
\text { NIV }\end{array}$ & $\begin{array}{l}\text { Age, mean } \pm \text { SD } \\
\text { or med (range) }\end{array}$ & NIV type & \multicolumn{3}{|c|}{$\begin{array}{l}\text { Length of hospital stay [mean } \pm \text { SD or med } \\
\text { (range)] }\end{array}$} & Mortality & & \\
\hline & & & & & & No ventilation & NIV & IMV & & & \\
\hline $\begin{array}{l}\text { Garcia Teresa } \\
\text { (60), 2017, } \\
\text { Spain }\end{array}$ & $\begin{array}{l}\text { P, Obs: } \\
\text { cross-sectional }\end{array}$ & $\mathrm{CHS}$ & $\mathrm{n} / \mathrm{a}$ & $\begin{array}{l}11.35 \text { ( } 5 \text { months to } \\
28.6 \text { years) }\end{array}$ & NIV & & $91 \pm 51$ days & $319 \pm 336$ days $^{\star \star}$ & $n=2$ infants & & \\
\hline
\end{tabular}

BPAP, bi-level positive airway pressure; IMV, invasive mechanical ventilation; NIV, non-invasive ventilation; Obs, observational study; OSA, obstructive sleep apnea; P, prospective; PRS, Pierre Robin sequence; R, retrospective; SMA1, spinal muscular atrophy type 1.

${ }^{*} p<0.05$.

${ }^{* *} p<0.01$.

${ }^{\dagger} p<0.001$. 


\begin{tabular}{|c|c|c|c|c|c|c|c|c|}
\hline \multirow[b]{2}{*}{ Study or Subgroup } & \multicolumn{2}{|c|}{ NIV Therapy } & \multicolumn{2}{|c|}{ Supportive Care } & \multirow[b]{2}{*}{ Weight } & \multirow{2}{*}{$\begin{array}{c}\text { Risk Ratio } \\
\text { M-H, Fixed, } 95 \% \mathrm{Cl}\end{array}$} & \multirow{2}{*}{\multicolumn{2}{|c|}{$\begin{array}{c}\text { Risk Ratio } \\
\text { M-H, Fixed, } 95 \% \mathrm{Cl}\end{array}$}} \\
\hline & Events & Total & Events & Total & & & & \\
\hline Bach 2002 & 2 & 33 & 7 & 7 & $14.3 \%$ & $0.08[0.02,0.26]$ & $\longrightarrow$ & \\
\hline Bach 2007 & 8 & 47 & 18 & 18 & $31.3 \%$ & $0.18[0.10,0.34]$ & $\longrightarrow$ & \\
\hline Gregoretti 2013 & 14 & 31 & 113 & 121 & $54.4 \%$ & $0.48[0.33,0.71]$ & & \\
\hline Total $(95 \% \mathrm{Cl})$ & & 111 & & 146 & $100.0 \%$ & $0.33[0.24,0.45]$ & & \\
\hline Total events & 24 & & 138 & & & & & \\
\hline $\begin{array}{l}\text { Heterogeneity: } \mathrm{Chi}^{2}= \\
\text { Test for overall effect }\end{array}$ & $\begin{array}{l}12.74, \mathrm{df}= \\
\mathrm{Z}=7.090\end{array}$ & $\begin{array}{l}2(\mathrm{P}= \\
2<0.00\end{array}$ & $\begin{array}{l}0.002) ; r^{2}= \\
0001)\end{array}$ & $4 \%$ & & & $\begin{array}{|cc|}0.02 & 0.1 \\
& \text { Favours NIV therapy }\end{array}$ & ${ }_{1}^{1} \quad 10 \quad 5$ \\
\hline
\end{tabular}

By contrast, extrapolation of outcomes for long-term NIV use in NMD and CNS disorders may be more challenging. The data relevant to long-term NIV use for NMD and CNS disorders are almost exclusively from two conditions: SMA1 and CHS. SMA1 is a progressively deteriorating disorder that is usually fatal during infancy. This contrasts with other NMD disorders presenting in infancy, such as congenital myopathy and congenital muscular dystrophy, which may have a better prognosis or steadier course $(7,58)$. The difference in prognoses of these conditions makes generalizing outcomes for NIV use in SMA1 to other NMD less appropriate. Similarly, CHS was the only CNS disorder for which data on long-term NIV use was available. NIV may be useful for other CNS disorders with accompanying respiratory compromise, such as congenital or acquired brain injury. Given the potentially unique physiology of CHS extrapolating the outcomes of NIV use for infants with CHS to other CNS conditions with different underlying respiratory pathophysiology may not be appropriate. Creation of national disease registries for infants and children using NIV will provide the opportunity to aggregate data on rare or minimally studied diseases and examine the use and outcomes of long-term NIV in these populations.

The outcomes that were reported in studies differed depending on the primary underlying disease category that was being examined. Studies of airway conditions predominantly reported on changes in respiratory parameters reported via polysomnography results and discontinuation of NIV. In addition, most studies reported short-term overnight polysomnography results; only one study had data on polysomnography results after long-term follow-up periods of NIV use in infants (29). Only one study on upper airway disorders reported on mortality outcomes (27) and none on hospitalization outcomes. Long-term outcomes, such as hospitalizations, intercurrent illness, growth and development, and quality of life warrant further study. Interestingly, studies on SMA1 predominantly reported on mortality and hospitalization outcomes, with only one study reporting on changes in respiratory parameters.

While the overall quality of the evidence available for the use of long-term NIV in infants is low to very low, there is a body of evidence that may help guide clinical practice. The reason for the low quality of the evidence included the study design and a high risk of bias due to the lack of blinding and randomization, and control for confounding variables. While these findings highlight the need for future studies of strong design and lower risk of bias, the available data still provide important information to inform treatment decisions for conditions where long-term NIV is being considered.

\section{Limitations of the Included Studies}

We identified a number of research gaps present in the studies included within this review. There was only one study that compared the efficacy of CPAP and BPAP ventilation in a cohort of infants (38). Similarly, while some studies reported mask complications $(9,21)$, only one compared the efficacy and practicality of different infant NIV masks (74). Only single studies were identified on the use of long-term NIV for infants with breath holding (42) and cardiac disease (66). Additionally, there were no studies on the clinical supports necessary for infants to be placed on NIV. It is important to know whether infants receive consultation and support from physicians, registered nurses, home care support, or a combination thereof, to determine whether a multidisciplinary NIV care plan is necessary for this population. The lack of comparison groups and/or homogeneity of outcomes reported precluded meta-analysis for most topics.

Additional issues relevant to long-term NIV use in infants that are not addressed in the current literature include: limitations in availability of masks and headgear; limitations in the availability of BPAP machines that are sufficiently sensitive to detect flow rates; the impact of NIV use on craniofacial growth and the impact of craniofacial growth on NIV use; comorbidities in infants using NIV; the impact of NIV on somatic growth and psychomotor development; and, most importantly, the impact of NIV use on quality of life for both infants and caregivers.

\section{Limitations of the Review}

Our review relied on the search methods and primary-level screening decisions of a scoping review on NIV in children with subsequent development of the research questions on NIV in infants. The methods to identify studies for the scoping review, however, were sufficiently inclusive to capture all relevant evidence on NIV in infants. We defined NIV for the scoping review on long-term NIV as breathing support outside the airway via an interface, consistent with the $\mathrm{MeSH}$ 
TABLE 5 | Assessment of risk of bias in studies synthesized in the systematic review on long-term non-invasive ventilation in infants using the Risk of Bias in Nonrandomized Studies of Interventions (ROBINS-I) tool (18).

\begin{tabular}{|c|c|c|c|c|c|c|c|}
\hline First author, year & Confounding & Selection & $\begin{array}{l}\text { Measurement of } \\
\text { intervention }\end{array}$ & Missing data & $\begin{array}{l}\text { Measurement of } \\
\text { outcomes }\end{array}$ & $\begin{array}{l}\text { Selection of } \\
\text { reported results }\end{array}$ & $\begin{array}{l}\text { Overall risk } \\
\text { of bias (RoB) } \\
\text { assessment }^{a}\end{array}$ \\
\hline \multicolumn{8}{|l|}{ Obstructive sleep apnea } \\
\hline Downey (20), 2000 & Moderate & Moderate & Serious & Serious & Serious & Serious & Serious \\
\hline Guilleminault (21), 1995 & Serious & Serious & Serious & Serious & Serious & Moderate & Serious \\
\hline Harrington (22), 2003 & Moderate & Moderate & Serious & Moderate & Moderate & Moderate & Serious \\
\hline Leonardis (23), 2013 & Moderate & Serious & Moderate & Serious & Serious & Moderate & Serious \\
\hline Liu (24), 2012 & Serious & Serious & Moderate & Moderate & Moderate & Serious & Serious \\
\hline McNamara (27), 1995 & Moderate & Moderate & Moderate & Serious & Moderate & Moderate & Serious \\
\hline McNamara (28), 1999a & Moderate & Moderate & Moderate & Moderate & Moderate & Moderate & Moderate \\
\hline McNamara (29), 1999b & Moderate & Moderate & Moderate & Moderate & Moderate & Moderate & Moderate \\
\hline Robison (10), 2013 & Moderate & Moderate & Serious & Serious & Serious & Moderate & Serious \\
\hline Rosen (30), 2010 & Moderate & Serious & Serious & Serious & Serious & Serious & Serious \\
\hline
\end{tabular}

\section{Pierre Robin sequence}

\begin{tabular}{|c|c|c|c|c|c|c|c|}
\hline Amaddeo (31), 2016 & Serious & Serious & Serious & Moderate & Serious & Moderate & Serious \\
\hline Kam (35), 2015 & Moderate & Moderate & Serious & Serious & Moderate & Moderate & Serious \\
\hline Leboulanger (36), 2010 & Moderate & Moderate & Serious & Moderate & Moderate & Moderate & Serious \\
\hline Goudy (34), 2017 & Serious & Serious & Serious & Moderate & Serious & Moderate & Serious \\
\hline \multicolumn{8}{|c|}{ Laryngo-tracheomalacia } \\
\hline Essouri (38), 2005 & Moderate & Moderate & Moderate & Moderate & Low & Low & Moderate \\
\hline Fauroux (39), 2001 & Moderate & Moderate & Moderate & Serious & Moderate & Moderate & Serious \\
\hline Shatz (40), 2004 & Moderate & Serious & Serious & Serious & Serious & Moderate & Serious \\
\hline Zwacka (41), 1997 & Serious & Serious & Serious & Serious & Serious & Serious & Serious \\
\hline
\end{tabular}

Spinal muscular atrophy type 1

\begin{tabular}{|c|c|c|c|c|c|c|c|}
\hline Bach (43), 2000 & Serious & Serious & Serious & Serious & Serious & Serious & Serious \\
\hline Bach (44), 2002 & Serious & Serious & Serious & Serious & Serious & Serious & Serious \\
\hline Bach (46), 2007 & Serious & Serious & Serious & Serious & Low & Moderate & Serious \\
\hline Birnkrant (48), 1998 & Serious & Serious & Serious & Moderate & Serious & Serious & Serious \\
\hline Chatwin (49), 2011 & Serious & Serious & Serious & Moderate & Moderate & Serious & Serious \\
\hline Gregoretti (51), 2013 & Moderate & Moderate & Moderate & Moderate & Moderate & Moderate & Moderate \\
\hline Lemoine (53), 2012 & Moderate & Serious & Serious & Moderate & Moderate & Moderate & Serious \\
\hline Ottonello (54), 2011 & Moderate & Serious & Serious & Moderate & Moderate & Moderate & Serious \\
\hline Vasconcelos (56), 2005 & Serious & Serious & Serious & Serious & Serious & Serious & Serious \\
\hline \multicolumn{8}{|c|}{ Congenital hypoventilation syndrome } \\
\hline Hartmann (61), 1994 & Serious & Serious & Serious & Serious & Serious & Serious & Serious \\
\hline Noyes (63), 1999 & Serious & Serious & Serious & Serious & Serious & Serious & Serious \\
\hline Ramesh (64), 2008 & Moderate & Serious & Serious & Moderate & Serious & Serious & Serious \\
\hline Tibballs (65), 2003 & Moderate & Serious & Serious & Moderate & Serious & Serious & Serious \\
\hline García Teresa (60), 2017 & Serious & Serious & Serious & Moderate & Serious & Serious & Serious \\
\hline Khayat (62), 2017 & Serious & Serious & Serious & Moderate & Serious & Moderate & Serious \\
\hline
\end{tabular}

Low risk of bias - study is comparable to a well performed randomized trial within that domain. Moderate risk of bias - study is sound for a non-randomized study, but is not considered comparable to a well performed randomized trial within that domain. Serious risk of bias - study has some important problems within that domain. Critical risk of biasthe study is too problematic in this domain to provide any useful evidence on the effects of intervention. No information - no information on which to base a judgment about risk of bias within that domain.

${ }^{a}$ Criteria set out by the ROBINS-I tool.

terminology for NIV and, therefore, included CPAP as well as BPAP. Some investigators, however, do not consider CPAP as a mode of NIV because it requires spontaneous breathing from the patient $(1,77)$. To address this concern, we reported the different ventilation types used by infants in the tables included in this review. Finally, we defined infants as ages $0-2$ years based on the Public Health Agency of Canada definition (14).
Some investigators may not agree with this definition, as the Centre for Disease Control defines infants as less than one year of age (78). Regardless of the definition used, it is still unclear whether there are differences in the outcomes of pediatric NIV with respect to age. Future work should consider whether infants represent a distinct group within children using longterm NIV. 
TABLE 6 | Quality assessment of outcomes of infants using long-term non-invasive ventilation using the Grading of Recommendations Assessment, Development and Evaluation criteria (19).

\begin{tabular}{|c|c|c|c|c|c|c|c|c|c|c|c|c|}
\hline \multirow{2}{*}{$\begin{array}{l}\text { Quality assessment } \\
\text { Number of studies }\end{array}$} & \multirow[b]{2}{*}{ Study design } & \multirow[b]{2}{*}{$\begin{array}{l}\text { Risk of } \\
\text { bias }^{a}\end{array}$} & \multirow[b]{2}{*}{ Inconsistency } & \multirow[b]{2}{*}{ Indirectness } & \multirow[b]{2}{*}{ Imprecision } & \multirow[b]{2}{*}{$\begin{array}{l}\text { Other } \\
\text { considerations }\end{array}$} & \multicolumn{2}{|c|}{ Number of patients } & \multicolumn{2}{|c|}{ Effect } & \multirow[t]{2}{*}{ Quality } & \multirow[t]{2}{*}{ Importance } \\
\hline & & & & & & & Intervention & Control & $\begin{array}{l}\text { Relative } \\
\text { (95\% Cl) }\end{array}$ & $\begin{array}{l}\text { Absolute } \\
(95 \% \mathrm{Cl})\end{array}$ & & \\
\hline
\end{tabular}

Obstructive sleep apnea

Changes in respiratory parameters: respiratory gases pre-NIV to post-NIV

\begin{tabular}{|c|c|c|c|c|c|c|c|c|c|c|}
\hline $\begin{array}{l}5(20,22,27-29) \\
3(10,23,24)\end{array}$ & $\begin{array}{l}\text { Observational } \\
\text { studies } \\
\text { Observational } \\
\text { studies }\end{array}$ & $\begin{array}{l}\text { Serious } \\
\text { Serious }\end{array}$ & $\begin{array}{l}\text { Not serious } \\
\text { Not serious }\end{array}$ & $\begin{array}{l}\text { Not serious } \\
\text { Not serious }\end{array}$ & $\begin{array}{l}\text { Not serious } \\
\text { Not serious }\end{array}$ & $\begin{array}{l}\text { None } \\
\text { None }\end{array}$ & $\begin{array}{c}53 \\
-\end{array}$ & $\begin{array}{l}53 \\
-\end{array}$ & $\begin{array}{l}\oplus \oplus \circ \bigcirc \\
\text { low } \\
\oplus \bigcirc \circ \bigcirc \\
\text { very low }\end{array}$ & $\begin{array}{l}\text { Important } \\
\text { Important }\end{array}$ \\
\hline \multicolumn{11}{|l|}{ Discontinuation of NIV } \\
\hline $5(20,21,27,28,30)$ & $\begin{array}{l}\text { Observational } \\
\text { studies }\end{array}$ & Serious & Not serious & Not serious & Not serious & None & - & - & $\begin{array}{l}\oplus \oplus \circ \bigcirc \\
\text { low }\end{array}$ & Important \\
\hline
\end{tabular}

Pierre Robin sequence

Changes in respiratory parameters: respiratory gases pre-NIV to post-NIV

\begin{tabular}{|c|c|c|c|c|c|c|c|c|c|c|}
\hline $2(31,36)$ & $\begin{array}{l}\text { Observational } \\
\text { study }\end{array}$ & Serious & Not serious & Not serious & Not serious & None & - & - & $\begin{array}{l}\oplus \circ \bigcirc \bigcirc \\
\text { very low }\end{array}$ & Important \\
\hline \multicolumn{11}{|c|}{ Discontinuation of NIV } \\
\hline $2(31,36)$ & $\begin{array}{l}\text { Observational } \\
\text { studies }\end{array}$ & Serious & Not serious & Not serious & Not serious & None & - & - & $\begin{array}{l}\oplus \circ \bigcirc \bigcirc \\
\text { very low }\end{array}$ & Important \\
\hline \multicolumn{11}{|c|}{ Length of hospitalization } \\
\hline $2(31,35)$ & $\begin{array}{l}\text { Observational } \\
\text { studies }\end{array}$ & Serious & Not serious & Not serious & Not serious & None & - & - & $\begin{array}{l}\oplus \circ \circ \bigcirc \\
\text { very low }\end{array}$ & Important \\
\hline \multicolumn{11}{|c|}{ Adherence } \\
\hline $2(31,36)$ & $\begin{array}{l}\text { Observational } \\
\text { studies }\end{array}$ & Serious & Not serious & Not serious & Not serious & None & - & - & $\begin{array}{l}\oplus \circ \bigcirc \bigcirc \\
\text { very low }\end{array}$ & Important \\
\hline
\end{tabular}

Laryngo-tracheomalacia

Changes in respiratory parameters: respiratory gases: supportive care vs. NIV

\begin{tabular}{|c|c|c|c|c|c|c|c|c|c|c|}
\hline $3(38,39,41)$ & $\begin{array}{l}\text { Observational } \\
\text { studies }\end{array}$ & Serious & Not serious & Not serious & Not serious & None & 24 & 24 & $\begin{array}{l}\oplus \oplus \circ \bigcirc \\
\text { low }\end{array}$ & Important \\
\hline \multicolumn{11}{|c|}{ Discontinuation of NIV } \\
\hline $2(39,40)$ & $\begin{array}{l}\text { Observational } \\
\text { studies }\end{array}$ & Serious & Not serious & Not serious & Not serious & None & - & - & $\begin{array}{l}\oplus \circ \circ \bigcirc \\
\text { very low }\end{array}$ & Important \\
\hline \multicolumn{11}{|c|}{$\begin{array}{l}\text { Benefit of NIV-improvement in } \\
\text { growth parameter(s) }\end{array}$} \\
\hline $1(39)$ & $\begin{array}{l}\text { Observational } \\
\text { study }\end{array}$ & Serious & Not serious & Not serious & Not serious & None & - & - & $\begin{array}{l}\oplus \circ \circ \bigcirc \\
\text { very low }\end{array}$ & Important \\
\hline \multicolumn{11}{|c|}{ Benefit of NIV-improvement in underlying condition(s) } \\
\hline $1(40)$ & $\begin{array}{l}\text { Observational } \\
\text { study }\end{array}$ & Serious & Not serious & Not serious & Not serious & None & - & - & $\begin{array}{l}\oplus \circ \bigcirc \bigcirc \\
\text { very low }\end{array}$ & Important \\
\hline
\end{tabular}


TABLE 6 | Continued

Quality assessment

Number of studies

Study design Risk of Inconsistency Indirectness Imprecision Other

Number of patients

Effect

Quality Importance

Adherence

1 (39) Risk of
bias $^{\mathrm{a}}$

considerations

Intervention Control Relative Absolute

$(95 \% \mathrm{Cl}) \quad(95 \% \mathrm{Cl})$

Observational Serious Not serious Not serious Not serious None

$\oplus \circ \bigcirc$ Important study

Not seious

(1)

very low

Spinal muscular atrophy type 1

Mortality: NIV vs. supportive care

$3(44,46,51)$

$\begin{array}{llllll}\text { Observational } & \text { Serious } & \text { Not serious } & \text { Not serious } & \text { Not serious } & \text { None } \\ \text { studies } & \text { Serious } & \text { Not serious } & \text { Not serious } & \text { Not serious } & \text { None } \\ \text { Observational } & & & & & \end{array}$

Observationa

studies

Hospitalization: per patient/per year

$\begin{array}{lllllll}3(43,46,51) & \text { Observational } & \text { Serious } & \text { Not serious } & \text { Not serious } & \text { Not serious } & \text { None } \\ 3(43,53,54) & \text { studies } & \text { Serious } & \text { Not serious } & \text { Not serious } & \text { Not serious } & \text { None } \\ & \begin{array}{l}\text { Observational } \\ \text { studies }\end{array} & & & & & \\ & & & & & \end{array}$

Benefit of NIV-improvement in growth parameter(s)

$3(44,46,54)$

Observational Serious Not serious Notserious Not serious None

Benefit of NIV-NIV facilitated

studies

(a) nons

extubation

$3(43,48,50)$

Observational Serious Not serious Not serious Not serious None

study

Changes in respiratory parameters:

respiratory gases

1 (55)

Observational Moderate Not serious Not serious Not serious None

study

24/111 138/146 RR $0.37 \quad 595$ fewer $\oplus \oplus \circ$ Ver

$\begin{array}{llllll}(21.6 \%) & (94.5 \%) & (0.25-0.54) & \text { per } 1000 & \text { low important }\end{array}$

$z=5.16 \quad$ (from $435 \quad \oplus \oplus \circ) \quad$ Very

$p<0.0001$ fewer low important

to 709

fewer)

Congenital hypoventilation syndrome

Changes in respiratory parameters: changes in respiratory gases post-NIV initiation

\begin{tabular}{|c|c|c|c|c|c|c|c|c|c|c|}
\hline $2(61,65)$ & $\begin{array}{l}\text { Observational } \\
\text { study }\end{array}$ & Serious & Not serious & Not serious & Not serious & None & - & - & $\begin{array}{l}\oplus \oplus \bigcirc \bigcirc \\
\text { very low }\end{array}$ & Important \\
\hline \multicolumn{11}{|c|}{ Discontinuation of NIV } \\
\hline $2(61,64)$ & $\begin{array}{l}\text { Observational } \\
\text { studies }\end{array}$ & Serious & Not serious & Not serious & Not serious & None & - & - & $\begin{array}{l}\oplus \bigcirc \bigcirc \bigcirc \\
\text { very low }\end{array}$ & Important \\
\hline \multicolumn{11}{|c|}{ Benefit of NIV-improvement in growth parameter(s) } \\
\hline $2(61,63)$ & $\begin{array}{l}\text { Observational } \\
\text { studies }\end{array}$ & Serious & Not serious & Not serious & Not serious & None & - & - & $\begin{array}{l}\oplus \bigcirc \bigcirc \bigcirc \\
\text { very low }\end{array}$ & Important \\
\hline
\end{tabular}

$\oplus \oplus \circ \bigcirc$ Very

low important

$\oplus \circ 00$ Important

$\oplus \oplus \bigcirc$ Important

low

$\oplus \circ \bigcirc \mathrm{Importan}$

very low

$\oplus \oplus \circ \bigcirc$ Important low 


\section{CONCLUSION}

This systematic review examines the use and outcomes of long-term NIV in infants across a range of respiratory and sleep disorders. Improvements in respiratory parameters and discontinuation from NIV due to improvement in underlying conditions have been shown for a broad range of upper airway disorders, such as OSA, PRS, and LTM, in infants. Long-term NIV use in infants with SMA1 decreased hospitalizations and prolonged survival compared to infants on supportive care. Infants with CHS may also show improvements in respiratory parameters after using NIV and potentially avoid tracheostomy. NIV appears to be a feasible method of providing long-term respiratory support for infants with a wide range of underlying conditions; however, several methodological weaknesses limit any strong categorical conclusions. The findings of this systematic review are relevant to a broad range of stakeholders and can be used to help guide clinicians on the use of long-term NIV in infants.

\section{AUTHOR CONTRIBUTIONS}

PB conceptualized and designed the review, assessed articles for inclusion, extracted and analyzed data, interpreted the data, drafted the initial manuscript, and completed all subsequent revisions until submission. MC conceptualized and designed the review, assessed articles for inclusion, verified data extraction, and critically reviewed the manuscript. RF developed the search strategy, carried out the literature searches, and critically reviewed the manuscript. MA and BA assessed articles for inclusion, and critically reviewed the manuscript. AK provided guidance on study design and critically reviewed the manuscript. CF provided guidance on study design and review methodology and critically reviewed the manuscript. JM conceptualized and designed the review, assessed articles for inclusion, verified data extraction, interpreted the data, and critically reviewed the manuscript. All authors reviewed the manuscript and approved the final manuscript for submission.

\section{ACKNOWLEDGMENTS}

The authors thank Dr. Meghan Sebastianski (Knowledge Translation Platform, Alberta SPOR SUPPORT Unit, University of Alberta) for her expertise and guidance on systematic review methodology.

\section{FUNDING}

The authors have no financial relationships relevant to this article to disclose. $\mathrm{PB}$ received salary support through a Pediatric Non-Invasive Ventilation studentship from the Department of Pediatrics, University of Alberta. The project was supported by a mini-grant from the Respiratory Health Strategic Clinical Network of Alberta Health Services. 


\section{REFERENCES}

1. Amin R, Sayal P, Syed F, Chaves A, Moraes TJ, MacLusky I. Pediatric long-term home mechanical ventilation: twenty years of follow-up from one Canadian center. Pediatr Pulmonol (2014) 49(8):816-24. doi:10.1002/ppul.22868

2. McDougall CM, Adderley RJ, Wensley DF, Seear MD. Long-term ventilation in children: longitudinal trends and outcomes. Arch Dis Child (2013) 98(9):660-5. doi:10.1136/archdischild-2012-303062

3. Amaddeo A, Moreau J, Frapin A, Khirani S, Felix O, Fernandez-Bolanos M, et al. Long term continuous positive airway pressure (CPAP) and noninvasive ventilation (NIV) in children: initiation criteria in real life. Pediatr Pulmonol (2016) 51(9):968-74. doi:10.1002/ppul.23416

4. Katz S, Selvadurai H, Keilty K, Mitchell M, MacLusky I. Outcome of non-invasive positive pressure ventilation in paediatric neuromuscular disease. Arch Dis Child (2004) 89(2):121-4. doi:10.1136/adc.2002.018655

5. Simonds AK, Ward S, Heather S, Bush A, Muntoni F. Outcome of paediatric domiciliary mask ventilation in neuromuscular and skeletal disease. Eur Respir J (2000) 16(3):476-81. doi:10.1034/j.1399-3003.2000.016003476.x

6. Jardine E, O’Toole M, Paton JY, Wallis C. Current status of long term ventilation of children in the United Kingdom: questionnaire survey. BMJ (1999) 318(7179):295-9. doi:10.1136/bmj.318.7179.295

7. Chatwin M, Tan HL, Bush A, Rosenthal M, Simonds AK. Long term non-invasive ventilation in children: impact on survival and transition to adult care. PLoS One (2015) 10(5):e0125839. doi:10.1371/journal.pone.0125839

8. Castro-Codesal ML, Dehaan K, Featherstone R, Bedi PK, Martinez Carrasco C, Katz SL, et al. Long-term non-invasive ventilation therapies in children: a scoping review. Sleep Med Rev (2018) 37:148-58. doi:10.1016/j. smrv.2017.02.005

9. Markstrom A, Sundell K, Stenberg N, Katz-Salamon M. Long-term non-invasive positive airway pressure ventilation in infants. Acta Paediatr (2008) 97(12):1658-62. doi:10.1111/j.1651-2227.2008.00990.x

10. Robison JG, Wilson C, Otteson TD, Chakravorty SS, Mehta DK. Analysis of outcomes in treatment of obstructive sleep apnea in infants. Laryngoscope (2013) 123(9):2306-14. doi:10.1002/lary.23685

11. MacLean JE, Fitzgerald DA, Waters KA. Developmental changes in sleep and breathing across infancy and childhood. Paediatr Respir Rev (2015) 16(4):276-84. doi:10.1016/j.prrv.2015.08.002

12. Iglowstein I, Jenni OG, Molinari L, Largo RH. Sleep duration from infancy to adolescence: reference values and generational trends. Pediatrics (2003) 111(2):302-7. doi:10.1542/peds.111.2.302

13. Marcus CL. Sleep-disordered breathing in children. Am J Respir Crit Care Med (2001) 164(1):16-30. doi:10.1164/ajrccm.164.1.2008171

14. Infancy (Birth - Two Years of Age). Public Health Agency of Canada (2016). Available from http://www.phac-aspc.gc.ca/hp-ps/dca-dea/stages-etapes/ childhood-enfance_0-2/index-eng.php

15. Moher D, Liberati A, Tetzlaff J, Altman DG, The PRISMA Group. Preferred reporting items for systematic reviews and meta-analyses: the PRISMA statement. BMJ (2009) 339:b2535. doi:10.1136/bmj.b2535

16. Bedi PK, Castro-Codesal ML, Featherstone R, AlBalawi MA, Alkhaledi B, Kozyrskyj A, et al. Long-term non-invasive ventilation in infants: a systematic review. PROSPERO 2016:CRD42016051302. (2016). Available from http:// www.crd.york.ac.uk/PROSPERO/display_record.asp?ID=CRD42016051302

17. Castro-Codesal ML, Featherstone R, Martinez Carrasco C, Katz SL, Chan EY, Bendiak GN, et al. Long-term non-invasive ventilation therapies in children: a scoping review protocol. BMJ Open (2015) 5(8):e008697. doi:10.1136/ bmjopen-2015-008697

18. Sterne JA, Hernán MA, Reeves BC, Savović J, Berkman ND, Viswanathan M, et al. ROBINS-I: a tool for assessing risk of bias in non-randomised studies of interventions. BMJ (2016) 355:i4919. doi:10.1136/bmj.i4919

19. Guyatt GH, Oxman AD, Vist GE, Kunz R, Falck-Ytter Y, Alonso-Coello P, et al. GRADE: an emerging consensus on rating quality of evidence and strength of recommendations. BMJ (2008) 336(7650):924-6. doi:10.1136/ bmj.39489.470347.AD

20. Downey R III, Perkin RM, MacQuarrie J. Nasal continuous positive airway pressure use in children with obstructive sleep apnea younger than 2 years of age. Chest (2000) 117(6):1608-12. doi:10.1378/chest.117.6.1608

21. Guilleminault C, Pelayo R, Clerk A, Leger D, Bocian RC. Home nasal continuous positive airway pressure in infants with sleep-disordered breathing. $J$ Pediatr (1995) 127(6):905-12. doi:10.1016/S0022-3476(95)70026-9
22. Harrington C, Kirjavainen T, Teng A, Sullivan CE. nCPAP improves abnormal autonomic function in at-risk-for-SIDS infants with OSA. J Appl Physiol (2003) 95(4):1591-7. doi:10.1152/japplphysiol.00354.2002

23. Leonardis RL, Robison JG, Otteson TD. Evaluating the management of obstructive sleep apnea in neonates and infants. JAMA Otolaryngol Head Neck Surg (2013) 139(2):139-46. doi:10.1001/jamaoto.2013.1331

24. Liu D, Zhou J, Liang X, Huang Z, Tan Z, Zhong J. Remote monitoring of home-based noninvasive ventilation in children with obstructive sleep apnea-hypopnea syndrome. Sleep Breath (2012) 16(2):317-28. doi:10.1007/ s11325-011-0516-y

25. Marcus CL, Ward SL, Mallory GB, Rosen CL, Beckerman RC, Weese-Mayer $\mathrm{DE}$, et al. Use of nasal continuous positive airway pressure as treatment of childhood obstructive sleep apnea. J Pediatr (1995) 127(1):88-94. doi:10.1016/ S0022-3476(95)70262-8

26. Massa F, Gonsalez S, Laverty A, Wallis C, Lane R. The use of nasal continuous positive airway pressure to treat obstructive sleep apnoea. Arch Dis Child (2002) 87(5):438-43. doi:10.1136/adc.87.5.438

27. McNamara F, Harris MA, Sullivan CE. Effects of nasal continuous positive airway pressure on apnoea index and sleep in infants. J Paediatr Child Health (1995) 31(2):88-94. doi:10.1111/j.1440-1754.1995.tb00753.x

28. McNamara F, Sullivan CE. Effects of nasal CPAP therapy on respiratory and spontaneous arousals in infants with OSA. J Appl Physiol (1999) 87(3):889-96. doi:10.1152/jappl.1999.87.3.889

29. McNamara F, Sullivan CE. Obstructive sleep apnea in infants and its management with nasal continuous positive airway pressure. Chest (1999) 116(1):10-6. doi:10.1378/chest.116.1.10

30. Rosen D. Some infants with Down syndrome spontaneously outgrow their obstructive sleep apnea. Clin Pediatr (Phila) (2010) 49(11):1068-71. doi:10.1177/0009922810378037

31. Amaddeo A, Abadie V, Chalouhi C, Kadlub N, Frapin A, Lapillonne A, et al. Continuous positive airway pressure for upper airway obstruction in infants with Pierre Robin sequence. Plast Reconstr Surg (2016) 137(2):609-12. doi:10.1097/01.prs.0000475799.07597.23

32. Cheng ATL, Corke M, Loughran-Fowlds A, Birman C, Hayward P, Waters KA. Distraction osteogenesis and glossopexy for Robin sequence with airway obstruction. ANZ J Surg (2011) 81(5):320-5. doi:10.1111/j.1445-2197.2010. 05588.x

33. Daniel M, Bailey S, Walker K, Hensley R, Kol-Castro C, Badawi N, et al. Airway, feeding and growth in infants with Robin sequence and sleep apnoea. Int J Pediatr Otorhinolaryngol (2013) 77(4):499-503. doi:10.1016/j. ijporl.2012.12.019

34. Goudy S, Jiramongolchai P, Chinnadurai S. Logistic regression analysis of Pierre Robin sequence patients requiring surgical intervention. Laryngoscope (2017) 127(4):945-9. doi:10.1002/lary.26143

35. Kam K, McKay M, MacLean J, Witmans M, Spier S, Mitchell I. Surgical versus nonsurgical interventions to relieve upper airway obstruction in children with Pierre Robin sequence. Can Respir J (2015) 22(3):171-5. doi:10.1155/2015/798076

36. Leboulanger N, Picard A, Soupre V, Aubertin G, Denoyelle F, Galliani E, et al. Physiologic and clinical benefits of noninvasive ventilation in infants with Pierre Robin sequence. Pediatrics (2010) 126(5):e1056-63. doi:10.1542/ peds.2010-0856

37. Muller-Hagedorn S, Buchenau W, Arand J, Bacher M, Poets CF. Treatment of infants with Syndromic Robin sequence with modified palatal plates: a minimally invasive treatment option. Head Face Med (2017) 13(1):4. doi:10.1186/ s13005-017-0137-1

38. Essouri S, Nicot F, Clément A, Garabedian EN, Roger G, Lofaso F, et al. Noninvasive positive pressure ventilation in infants with upper airway obstruction: comparison of continuous and bilevel positive pressure. Intensive Care Med (2005) 31(4):574-80. doi:10.1007/s00134-005-2568-6

39. Fauroux B, Pigeot J, Polkey MI, Roger G, Boulé M, Clément A, et al. Chronic stridor caused by laryngomalacia in children: work of breathing and effects of noninvasive ventilatory assistance. Am J Respir Crit Care Med (2001) 164(10 Pt 1):1874-8. doi:10.1164/ajrccm.164.10.2012141

40. Shatz A, Goldberg S, Picard E, Kerem E. Pharyngeal wall collapse and multiple synchronous airway lesions. Ann Otol Rhinol Laryngol (2004) 113(6):483-7. doi:10.1177/000348940411300613

41. Zwacka G, Scholle S, Kemper G, Rieger B. Nasal CPAP therapy for infants with congenital stridor. Sleep Breath (1997) 2(4):85-97. 
42. Guilleminault C, Huang Y-S, Chan A, Hagen CC. Cyanotic breath-holding spells in children respond to adenotonsillectomy for sleep-disordered breathing. J Sleep Res (2007) 16(4):406-13. doi:10.1111/j.1365-2869.2007.00605.x

43. Bach JR, Niranjan V, Weaver B. Spinal muscular atrophy type 1: a noninvasive respiratory management approach. Chest (2000) 117(4):1100-5. doi:10.1378/ chest.117.4.1100

44. Bach JR, Baird JS, Plosky D, Navado J, Weaver B. Spinal muscular atrophy type 1: management and outcomes. Pediatr Pulmonol (2002) 34(1):16-22. doi:10.1002/ppul.10110

45. Bach JR, Bianchi C. Prevention of pectus excavatum for children with spinal muscular atrophy type 1. Am J Phys Med Rehabil (2003) 82(10):815-9. doi:10.1097/01.PHM.0000083669.22483.04

46. Bach JR, Saltstein K, Sinquee D, Weaver B, Komaroff E. Long-term survival in Werdnig-Hoffmann disease. Am J Phys Med Rehabil (2007) 86(5):339-345; quiz 346-338, 379. doi:10.1097/PHM.0b013e31804a8505

47. Barnérias C, Quijano S, Mayer M, Estournet B, Cuisset JM, Sukno S, et al. [Multicentric study of medical care and practices in spinal muscular atrophy type 1 over two 10-year periods]. Arch Pediatr (2014) 21(4):347-54. doi:10.1016/j.arcped.2014.01.017

48. Birnkrant DJ, Pope JF, Martin JE, Repucci AH, Eiben RM. Treatment of type I spinal muscular atrophy with noninvasive ventilation and gastrostomy feeding. Pediatr Neurol (1998) 18(5):407-10. doi:10.1016/S0887-8994(97) 00227-0

49. Chatwin M, Bush A, Simonds AK. Outcome of goal-directed non-invasive ventilation and mechanical insufflation/exsufflation in spinal muscular atrophy type I. Arch Dis Child (2011) 96(5):426-32. doi:10.1136/adc.2009.177832

50. Ednick M, Wong B, Inge T, Knue M, Kalra M. Post-operative respiratory outcomes using a standard extubation protocol after elective gastrostomy tube placement in pediatric patients with spinal muscular atrophy type 1.J Pe1diatr Neurol (2008) 6(3):249-52. doi:10.1055/s-0035-1557467

51. Gregoretti C, Ottonello G, Chiarini Testa MB, Mastella C, Ravà L, Bignamini E, et al. Survival of patients with spinal muscular atrophy type 1. Pediatrics (2013) 131(5):e1509-14. doi:10.1542/peds.2012-2278

52. Ioos C, Leclair-Richard D, Mrad S, Barois A, Estournet-Mathiaud B. Respiratory capacity course in patients with infantile spinal muscular atrophy. Chest (2004) 126(3):831-7. doi:10.1378/chest.126.3.831

53. Lemoine TJ, Swoboda KJ, Bratton SL, Holubkov R, Mundorff M, Srivastava R. Spinal muscular atrophy type 1: are proactive respiratory interventions associated with longer survival? Pediatr Crit Care Med (2012) 13(3):e161-5. doi:10.1097/PCC.0b013e3182388ad1

54. Ottonello G, Mastella C, Franceschi A, Bosticco D, Wolfler A, Pedemonte $\mathrm{M}$, et al. Spinal muscular atrophy type 1: avoidance of hospitalization by respiratory muscle support. Am J Phys Med Rehabil (2011) 90(11):895-900. doi:10.1097/PHM.0b013e318232883a

55. Petrone A, Pavone M, Testa MBC, Petreschi F, Bertini E, Cutrera R. Noninvasive ventilation in children with spinal muscular atrophy types 1 and 2. Am J Phys Med Rehabil (2007) 86(3):216-21. doi:10.1097/PHM.0b013e31802ef774

56. Vasconcelos M, Fineza I, Felix M, Estevao MH. Spinal muscular atrophy - noninvasive ventilatory support in pediatrics. Rev Port Pneumol (2005) 11(5):443-55. doi:10.1016/S0873-2159(15)30520-1

57. Afsharpaiman S, Sillence DO, Sheikhvatan M, Ault JE, Waters K. Respiratory events and obstructive sleep apnea in children with achondroplasia: investigation and treatment outcomes. Sleep Breath (2011) 15(4):755-61. doi:10.1007/ s11325-010-0432-6

58. Han YJ, Park JD, Lee B, Choi YH, Suh DI, Lim BC, et al. Home mechanical ventilation in childhood-onset hereditary neuromuscular diseases: 13 years' experience at a single center in Korea. PLoS One (2015) 10(3):e0122346. doi:10.1371/journal.pone. 0122346

59. Wood L, Cordts I, Atalaia A, Marini-Bettolo C, Maddison P, Phillips M, et al. The UK myotonic dystrophy patient registry: facilitating and accelerating clinical research. J Neurol (2017) 264(5):979-88. doi:10.1007/s00415-017-8483-2

60. García Teresa MA, Porto Abal R, Rodríguez Torres S, García Urabayen D, García Martínez S, Trang H, et al. [Spanish patients with central hypoventilation syndrome included in the European Registry. The 2015 data]. An Pediatr (2017) 86(5):255-63. doi:10.1016/j.anpedi.2016.05.008

61. Hartmann H, Jawad MH, Noyes J, Samuels MP, Southall DP. Negative extrathoracic pressure ventilation in central hypoventilation syndrome. Arch Dis Child (1994) 70(5):418-23. doi:10.1136/adc.70.5.418
62. Khayat A, Medin D, Syed F, Moraes TJ, Bin-Hasan S, Narang I, et al. Intelligent volume-assured pressured support (iVAPS) for the treatment of congenital central hypoventilation syndrome. Sleep Breath (2017) 21(2):513-9. doi:10.1007/s11325-017-1478-5

63. Noyes J, Hartmann H, Samuels M, Southall D. The experiences and views of parents who care for ventilator-dependent children. J Clin Nurs (1999) 8(4):440-50. doi:10.1046/j.1365-2702.1999.00258.x

64. Ramesh P, Boit P, Samuels M. Mask ventilation in the early management of congenital central hypoventilation syndrome. Arch Dis Child Fetal Neonatal Ed (2008) 93(6):F400-3. doi:10.1136/adc.2008.139931

65. Tibballs J, Henning RD. Noninvasive ventilatory strategies in the management of a newborn infant and three children with congenital central, hypoventilation syndrome. Pediatr Pulmonol (2003) 36(6):544-8. doi:10.1002/ppul.10392

66. Bunn HJ, Roberts P, Thomson AH. Noninvasive ventilation for the management of pulmonary hypertension associated with congenital heart disease in children. Pediatr Cardiol (2004) 25(4):357-9. doi:10.1007/s00246-003-0501-8

67. Adeleye A, Ho A, Nettel-Aguirre A, Buchhalter J, Kirk V. Noninvasive positive airway pressure treatment in children less than 12 months of age. Can Respir $J$ (2016) 2016:7654631. doi:10.1155/2016/7654631

68. Bertrand P, Fehlmann E, Lizama M, Holmgren N, Silva M, Sanchez I. [Home ventilatory assistance in Chilean children: 12 years' experience]. Arch Bronconeumol (2006) 42(4):165-70. doi:10.1157/13086621

69. Fauroux B, Lavis JF, Nicot F, Picard A, Boelle PY, Clément A, et al. Facial side effects during noninvasive positive pressure ventilation in children. Intensive Care Med (2005) 31(7):965-9. doi:10.1007/s00134-005-2669-2

70. Kherani T, Sayal A, Al-Saleh S, Sayal P, Amin R. A comparison of invasive and noninvasive ventilation in children less than 1 year of age: a long-term follow-up study. Pediatr Pulmonol (2016) 51(2):189-95. doi:10.1002/ppul.23229

71. Koontz KL, Slifer KJ, Cataldo MD, Marcus CL. Improving pediatric compliance with positive airway pressure therapy: the impact of behavioral intervention. Sleep (2003) 26(8):1010-5. doi:10.1093/sleep/26.8.1010

72. Machaalani R, Evans CA, Waters KA. Objective adherence to positive airway pressure therapy in an Australian paediatric cohort. Sleep Breath (2016) 20(4):1327-36. doi:10.1007/s11325-016-1400-6

73. Nathan AM, Loo HY, de Bruyne JA, Eg KP, Kee SY, Thavagnanam S, et al. Thirteen years of invasive and noninvasive home ventilation for children in a developing country: a retrospective study. Pediatr Pulmonol (2017) 52(4):500-7. doi:10.1002/ppul.23569

74. Ramirez A, Delord V, Khirani S, Leroux K, Cassier S, Kadlub N, et al. Interfaces for long-term noninvasive positive pressure ventilation in children. Intensive Care Med (2012) 38(4):655-62. doi:10.1007/s00134-012-2516-1

75. Zhou J, Liu DB, Zhong JW, Huang ZY, Qiu SY, Zhou YP, et al. Feasibility of a remote monitoring system for home-based non-invasive positive pressure ventilation of children and infants (Provisional abstract). Int $J$ Pediatr Otorhinolaryngol (2012) 76(12):1737-40. doi:10.1016/j.ijporl.2012.08.012

76. Katz ES, Mitchell RB, D’Ambrosio CM. Obstructive sleep apnea in infants. Am J Respir Crit Care Med (2012) 185(8):805-16. doi:10.1164/ rccm.201108-1455CI

77. Amaddeo A, Caldarelli V, Fernandez-Bolanos M, Moreau J, Ramirez A, Khirani S, et al. Polygraphic respiratory events during sleep in children treated with home continuous positive airway pressure: description and clinical consequences. Sleep Med (2015) 16(1):107-12. doi:10.1016/j.sleep.2014.07.030

78. Centres for Disease Control and Prevention. Child Development: Infants (0-1 Year of Age). (2017). Available from: https://www.cdc.gov/ncbddd/childdevelopment/positiveparenting/infants.html

Conflict of Interest Statement: The authors declare that the research was conducted in the absence of any commercial or financial relationships that could be construed as a potential conflict of interest.

The reviewer MP and handling editor declared their shared affiliation.

Copyright (c) 2018 Bedi, Castro-Codesal, Featherstone, AlBalawi, Alkhaledi, Kozyrskyj, Flores-Mir and MacLean. This is an open-access article distributed under the terms of the Creative Commons Attribution License (CC BY). The use, distribution or reproduction in other forums is permitted, provided the original author(s) and the copyright owner are credited and that the original publication in this journal is cited, in accordance with accepted academic practice. No use, distribution or reproduction is permitted which does not comply with these terms. 